\title{
PROTOTYPING CONVENTIONALLY WOUND HIGH-FREQUENCY MAGNETIC SENSORS FOR ITER
}

\author{
D. TESTA, ${ }^{*}$ M. TOUSSAINT, R. CHAVAN, A. ENCHEVA, $\dagger$ J. B. LISTER, J-M. MORET, \\ and F. SANCHEZ \\ Centre de Recherches en Physique des Plasmas, Ecole Polytechnique Fédérale de Lausanne (CRPP-EPFL) \\ Association EURATOM-Confédération Suisse, CH-1015 Lausanne, Switzerland
}

Received October 27, 2010

Accepted for Publication May 31, 2011

The high-frequency (HF) magnetic sensors for ITER are currently based on a conventional, Mirnov-type pickup coil, with an effective area in the range $0.03<(N A)_{E F F}$ $\left(\mathrm{m}^{2}\right)<0.1$; the sensor is required to provide measurements of magnetic instabilities with magnitude around $\left|\delta B / B_{\theta}\right| \sim 10^{-4}$ in the $10-\mathrm{kHz}$ to $2-\mathrm{MHz}$ frequency range. The physical, mechanical, and electrical properties of one representative ITER HF pickup coil design have been analyzed with particular attention to the manufacturing and assembly process for the winding pack, as its integrity was found to be of concern when performing a coupled electromagnetic, structural, and thermal analysis of the sensor. Three different options for the guiding grooves in that design have been tested, using copper and tungsten for the winding pack, but none of them has been convincing enough due to the likelihood of breakages of the thin grooving and of the tungsten wire itself. Hence, alternative designs still based on a conventional Mirnovtype pickup coil have been explored, and a nonconventional Mirnov-type pickup coil was produced using direct laser cutting of a Type 316 stainless steel hollow tube, avoiding the difficulties encountered during the winding operations for conventional Mirnov-type sensors. This process of manufacturing appears to be acceptable for HF magnetic sensors of Mirnov-type design in ITER, and it is recommended for future prototyping studies, as the effective area of our first prototype, $(N A)_{E F F} \sim 0.01 \mathrm{~m}^{2}$, was well below the ITER requirement. The electrical characteristics and the frequency response of all these prototypes were evaluated up to $8 \mathrm{MHz}$, with the results in good agreement with model calculations. The conventional Mirnov-type prototypes behave as expected in terms of their main electrical properties and should satisfy the present measurement performance requirements. Finally, a direct measurement of the effective area of these sensors has shown that the geometrical value is a sufficiently correct estimate of its actual value at low frequencies $(<10 \mathrm{kHz})$ when the winding pack closely follows the nominal shape of the coil itself.

KEYWORDS: high-frequency magnetic sensors, ITER magnetic diagnostic system

\section{INTRODUCTION}

The high-frequency (HF) magnetic sensor for ITER is presently assumed to be a conventional, Mirnov-type pickup coil, i.e., a metallic wire wound on a ceramic insulating former. The nominal engineering specifications for this sensor and the measurement requirements for the HF magnetic diagnostic system as a whole have been presented in several ITER documents, an overview

\footnotetext{
*E-mail: duccio.testa@epfl.ch

$\dagger$ Current address: ITER Organization, F-13108 Saint-Paul-lezDurance, France
}

of which is available as Ref. $1 .^{\text {a }}$ Collating the evolving information from these sources, the specifications call

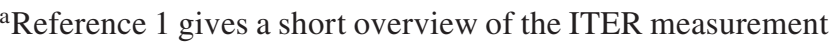
requirements and engineering specifications for the HF magnetic sensor and the overall diagnostic system. For a more complete overview of these requirements, see the ITER Design Description Document DDD 5.5.A, ITER document reference N55DDD101-06-12W0.3. See also the various presentations made at the ITER diagnostics review meetings on July 9-13, 2007. See also several presentations made at meetings of the International Tokamak Physics Activity (ITPA) working group on diagnostics. All these documents are available by request to duccio.testa@epfl.ch.
} 
for an inductive sensor with an effective area $(N A)_{E F F}$ in the range $0.03<(N A)_{E F F}\left(\mathrm{~m}^{2}\right)<0.1$. The sensor should measure HF magnetic instabilities with amplitude in the range $10^{-4}<\left|\delta B / B_{\theta}\right|<10^{-2}$ at the position of the sensors, where $B_{\theta} \sim 1 \mathrm{~T}$ is the equilibrium poloidal magnetic field. The required frequency range is between $10 \mathrm{kHz}$ and $2 \mathrm{MHz}$, although the lower end of this frequency range may have to be brought down to below $1 \mathrm{kHz}$ for some of these sensors, depending on their possible use as backup measurement for equilibrium reconstruction. Finally, this diagnostic system shall recognize toroidal mode numbers $n$ up to $|n|<50$ in all ITER main operating scenarios. The HF magnetic sensors are to be installed primarily in the back face of some of the blanket modules within a cutout of the order of $50 \times 50 \times 50 \mathrm{~mm}$. A secondary location has recently been considered, namely the continuous, but thinner-than-10-mm-depth gap between the back face of all the blanket modules and the inner face of the ITER double vacuum vessel wall. Additional sensors may also be located along the horizontal and vertical edges of selected equatorial ports, where provisions for this are already incorporated in the corresponding ports' design. These different requirements and restrictions provide the reference background for any appropriate design of these sensors.

A wealth of literature is available describing HF magnetic diagnostic systems in existing fusion devices, together with the generally accepted requirements for the measurement of HF instabilities in present and future burning-plasma experiments, and an overview is proposed as Ref. 2. ${ }^{\mathrm{b}}$ Distilling from all this available documentation, the target design parameters, such as effective area and electrical properties, of the HF ITER magnetic sensors should allow measurements of magnetohydrodynamic (MHD) instabilities in the frequency range between $10 \mathrm{kHz}$ and at least $500 \mathrm{kHz}$, but not necessarily above $1 \mathrm{MHz}$, hence, less demanding than the 2-MHz nominal requirement given in Ref. 1; detected instability magnitudes should be as low as practically possible, in the range $\left|\delta B / B_{\theta}\right| \sim 10^{-6}$ or lower, which is below the nominal requirement given in Ref. 1 . In fact, a larger value $\left|\delta B / B_{\theta}\right| \sim 10^{-4}$ is considered to be of a similar order to the mode amplitude threshold expected to cause stochastic fast ion transport ${ }^{3}$ and therefore not appropriate for detecting any onset of MHD activity. In current experiments, values of $|\delta B|$ of the order of a few milligauss are routinely measured, such as on JET (Ref. 4),

\footnotetext{
${ }^{\mathrm{b}}$ See also several presentations made at meetings of the ITPA working groups on diagnostics, energetic particles, and MHD (all these documents are available by request to duccio.testa@epfl.ch). For a general overview of the available literature on magnetic diagnostic systems see also the webpage http://crpp.epfl.ch/itermag/index.php/ SCI:Bibliography (updated at regular intervals, access available by request to duccio.testa@epfl.ch).
}

ASDEX-Upgrade (Ref. 5), MAST (Ref. 6), DIII-D (Ref. 7), and JT60U (Ref. 8).

An effective area $(N A)_{E F F}$ in the range from 0.05 to $0.10 \mathrm{~m}^{2}$ (as in Ref. 1) appears clearly appropriate to achieve these measurements, but there is an apparent discrepancy between the required $(N A)_{E F F}$ and the assumed $\left|\delta B / B_{\theta}\right|$ in Ref. 1 , and it is the current ITER design parameter $\left|\delta B / B_{\theta}\right| \sim 10^{-4}$ that appears to have been incorrectly specified. ${ }^{9}$ [In this respect, we note that during the recent conceptual design review of the HF magnetic diagnostic system in ITER (held from Nov. 30 to Dec. 2, 2010), a reference value for the measurable $|\delta B|$ to be in the range $10^{-6}<\left|\delta B / B_{\theta}\right|<10^{-2}$ has been proposed $\left.{ }^{10}\right]$. Finally, on the basis of results from existing tokamaks and predictions for ITER, this HF diagnostic system shall have the capability of recognizing toroidal mode numbers from up to $|n|=20$ to up to $|n|=30$ (Refs. 10 and 11) - somewhat less demanding than the $|n|=50$ requirement (Ref. 1) —and to recognize poloidal mode numbers $m$ from up to $|m|=30$ to up to $|m|=$ 40 (Ref. 11). Assuming a resonant magnetic surface with $q=m / n=2$, the requirements are consistent with measuring low- $m /$ low- $n$ neoclassical tearing modes..$^{10,11}$ The requirements on the diagnostic system and the sensors themselves can be separated into sensor design requirements and sensor layout requirements. The latter has been discussed in detail elsewhere. ${ }^{12}$

The previous development of the HF Mirnov-type pickup coil design was based on a sensor in use on ASDEX-Upgrade (Ref. 13 ${ }^{\mathrm{c}}$ ). The ITER concept, ${ }^{\mathrm{d}}$ which was adapted from the original ASDEX design to maximize its effective area while keeping a similar volume occupation, had not been manufactured before this prototyping. The goal of the initial prototyping tests was to demonstrate a manufacturing design satisfying three objectives: first, sufficiently strong to withstand the thermal and mechanical stress induced by plasma operation over the ITER machine lifetime ${ }^{14 \mathrm{e}}$; second, easy to manufacture by conventional machining processes; and third, easy to assemble and mount on in-vessel support structures, as there would clearly be more than one component in the final assembly.

Given the harsh environment of ITER, and before actual prototyping work started, we performed an initial evaluation of the design using a finite element method (FEM) analysis, in which electromagnetic, structural, and thermal studies were coupled together as far as practical.

\footnotetext{
${ }^{\mathrm{c}}$ Reference 13 is provided courtesy of G. Vayakis, ITER Organization.

${ }^{\mathrm{d}}$ ITER Organization, original drawings for the sensor design and in-vessel layout of the HF magnetic diagnostic system and all supplemental technical information provided courtesy of G. Vayakis, A. Encheva, and C. Walker (drawing series 55.0056.0001.2D.NNN).

${ }^{\mathrm{e}}$ Reference 14 provides a general overview of the ITER environmental condition and its operating regimes.
} 
This allowed us to identify weaker parts in the assembly, which could then be studied in detail in the prototyping. In the case of a wound-wire Mirnov-type pickup coil, the fact that such sensors have been in use in fusion experiments for almost 50 years already indicates that there is no obvious problem per se in this conceptual design. The first and most important item to be assessed was therefore the manufacturing and assembly of the winding pack given the specific design choices made for the particular sensor being tested.

This assembly study was performed effectively by using a material for the insulating former that is easier and cheaper to work on than the ceramics currently needed when manufacturing inductive magnetic sensors for fusion experiments (Shapal-M, alumina, boron oxide, and zirconium oxide were considered) and adopting the wire material foreseen for ITER, namely, copper or tungsten. Moreover, provided the material selected for the insulating former has similar electrical properties to those of the ceramics being considered, the sensor's electrical properties can be analyzed to verify whether these satisfy the measurement requirements. Using the cheaper and quicker material, we were able to prepare and test different prototypes with minor variations around the conceptual design for the insulating former, to assess possible problems in the assembly of the winding pack. This so-called "rapid prototyping" (RP) technology was therefore selected to produce the insulating former in polyamide 12 (PA12) for this first set of tasks. Successful initial tests should allow us to move with greater confidence toward preseries prototypes made using the ceramic materials cited, to evaluate the feasibility of the manufacturing processes for this design. Once the electrical properties are proven to be satisfactory and the ease of manufacture of a certain design is proven with the intended materials, it will be essential to run thermal, outgassing, and nuclear irradiation tests to qualify the sensor design for use in ITER.

The work described in this paper essentially reports on the first aspects of these prototyping tasks, namely, the FEM analysis, testing the assembly of the winding pack, and measuring and interpreting the electrical properties of the sensors. The starting point for this study (i.e., the reference design) did not allow a particularly easy and reliable assembly of the winding pack. This led to a design revision of this sensor. In parallel to this, we also started exploring two different strategies for manufacturing an appropriate HF magnetic sensor, based on the laser-cutting technique and on the low-temperature co-fired ceramic technology. The thermal and outgassing properties of the various conventional prototypes were not analyzed in this work due to the use of the RP technology. Conversely, the electrical properties of all these prototypes were analyzed by means of direct measurements of the transfer function of the pickup coil, including its resonant frequency, bandwidth, and effective area, and by modeling the equivalent circuit.
After this introduction and a brief review of the current design of the ITER HF Mirnov-type pickup coil, given in Sec. II, this paper is structured along three main subtopics. Section III presents the results of the FEM analysis performed on this design, which was used as one of the bases for our prototyping tests. The design and construction of different variants for the prototypes for this sensor, ensuing from the main results of the FEM analysis, are presented in Sec. IV, including a brief description of the RP technology. Section IV focuses on the results of the winding tests performed using a copper wire and a tungsten wire and on the proposed improvements in the manufacturing and winding processes for the ITER HF pickup coil of conventional design. These tests led to developing an alternative manufacturing process based on the laser-cutting technique, aimed at avoiding the difficulties encountered with conventional winding methods, also presented in Sec. IV. Section V presents the measurements and analysis of the electrical characteristics (resonance frequency, bandwidth, equivalent circuit model, and effective area) of these prototypes. Section VI gives a short summary of this work, together with an outlook toward future research and development (R\&D) and prototyping activities that we believe are needed to finalize the design for the ITER HF magnetic sensor. Finally, the Appendix presents the main aspects of the mathematical background required for the numerical determination of the electrical characteristics of the sensors.

(b)

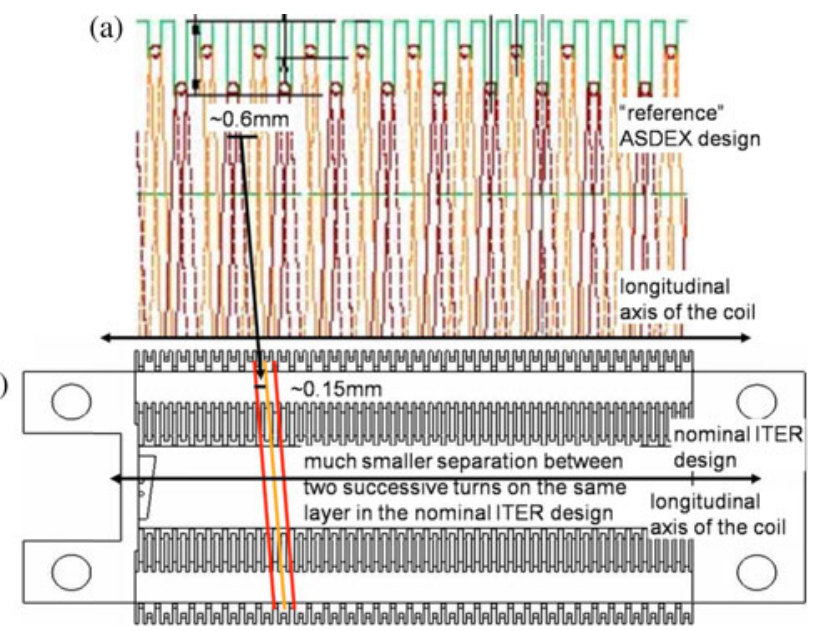

Fig. 1. A schematic drawing that illustrates graphically the main conceptual difference between the ASDEX-Upgrade and the ITER design of the HF Mirnov-type pickup coil: In the ASDEX-Upgrade design (shown here in an enlarged view to better illustrate its main features, i.e., with the line indicating the "longitudinal axis" drawn at half the coil radius), the separation between the two closest turns (one in the bottom layer, one in the top layer) is of the order of $\sim 0.6 \mathrm{~mm}$, whereas it is only $\sim 0.15 \mathrm{~mm}$ in the ITER design. 


\section{THE ASSUMED REFERENCE DESIGN OF THE ITER HF PICKUP COIL}

The main conceptual difference between the ASDEX-Upgrade and the basic features of the various phases of the ITER design (referred to in what follows simply as "the ITER design") has to do with the assembly of the winding pack, and this is sketched schemati- cally in Fig. 1. Both designs use two superposed concentric layers wound on a helicoidal path along the coil longitudinal axis, but in the ASDEX-Upgrade design (Fig. 1a), the helicoidal pitch is larger than in the ITER design (Fig. 1b). This modification doubles the effective area $(N A)_{E F F}$ of the ITER design while essentially keeping the same overall dimensions. However, this also means that the separation between the two closest turns

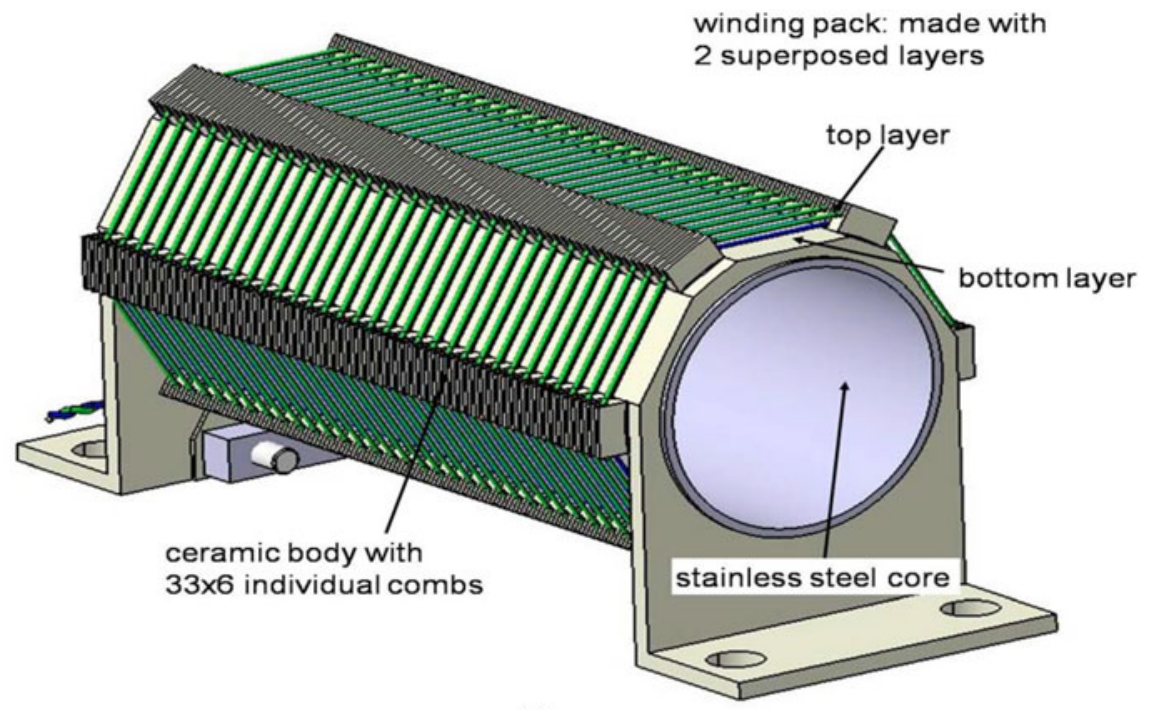

(a)

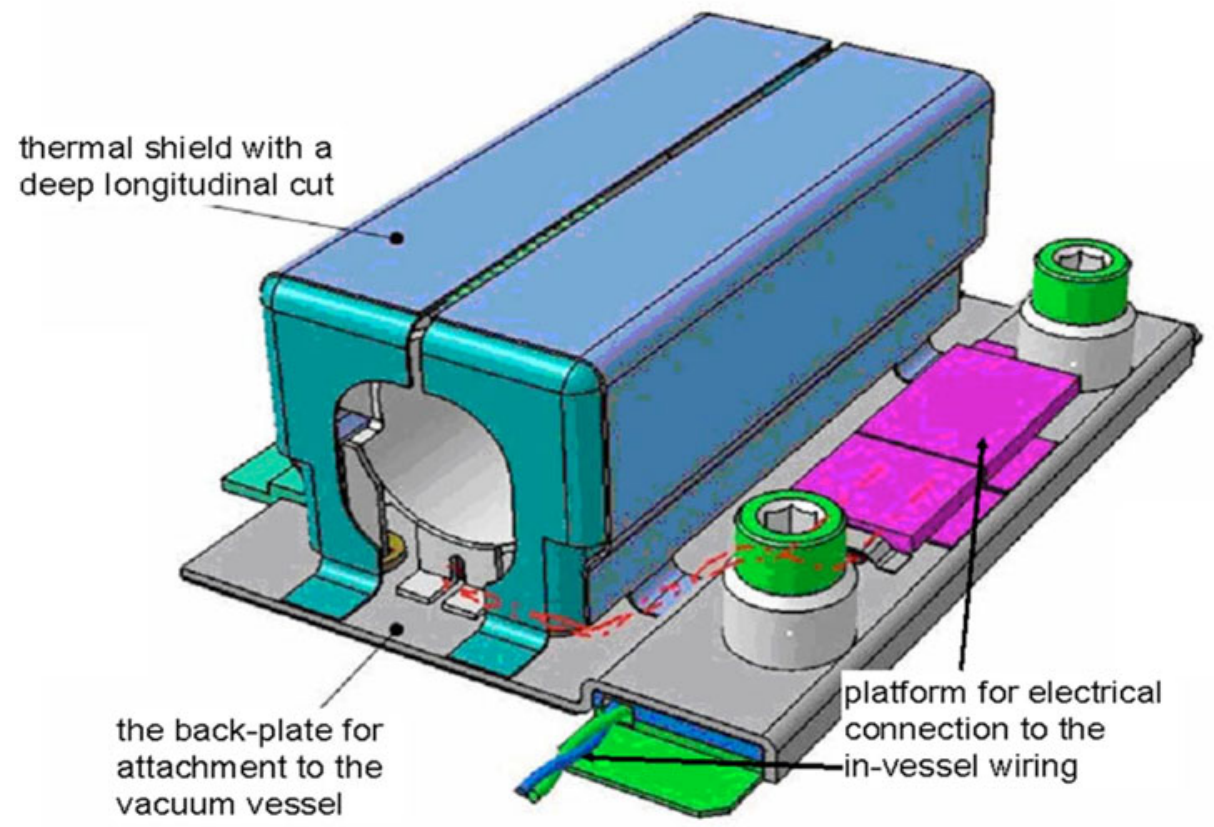

(b)

Fig. 2. ITER original (Mirnov-type) pickup coil assembly: the detailed view (a), showing the SS-316 core (in this case without a longitudinal cut) and the individual combs used to guide the wire and the winding pack, and the overall assembly (b), showing the thermal shield (with a longitudinal cut to allow for flux penetration), the back plate attachment, and the platform for connection to the in-vessel wiring. 
(one in the bottom layer, one in the top layer) is of the order of $\sim 0.6 \mathrm{~mm}$ in the ASDEX-Upgrade design, but only of $\sim 0.15 \mathrm{~mm}$ in the ITER design; i.e., there is a much thinner edge in the ceramic insulating former between these two turns.

Figure 2 illustrates the main features of the ITER design for the HF Mirnov-type pickup coil. This sensor is made with 33 turns each on two separate layers (Fig. 2a), a total of 66 turns wound over a hollow hexagonal ceramic body. This ITER design body is intended to be manufactured as a set of thin disks, each made of six individual "combs," easy to manufacture but individually weaker and requiring additional fixings to be kept in position during the assembly of the winding pack. Alternatively, this body could be made as a single component to have a much stronger part, on which sections are effectively "carved out" using some machining or molding processes, so that these sections will in fact act as the individual wire spacers. A Type 316 stainless steel (SS316) core mechanically supports the ceramic body, which is mounted on it by sprung compression-hence the individual wire spacers (the combs) - and also improves thermal conduction. This part is often called the "bobbin." It is the combination of tension in the wire and the spring-like compression from the bobbin that must hold the ceramic combs in position, but this can only occur upon completion of the assembly of the winding pack.
Hence, there is a delicate phase during assembly in which all components are loose.

Two design options were considered for the bobbin: to be made with or without a longitudinal cut. The need for basic mechanical strength, support, and improved frequency response (leading to a core without a cut) is traded off against the risk of circulating currents in the pickup coil assembly (which would not appear if the core had a longitudinal cut), since they would reduce the effective area for frequencies above a certain cutoff value. The diameter of the bobbin is intended to be slightly smaller than that of the ceramic body so that the bobbin can just slide inside the ceramic body at room temperature. Since the coefficient of thermal expansion (CTE) of the bobbin is larger than those of the proposed ceramics, there will be no problem with the differential thermal expansion between the bobbin and the individual components of the ceramic body when the assembly is mounted on the ITER vacuum vessel, since the essentially homogenous sliding compression stress induced by this differential thermal expansion is well below the typical limit for such ceramic materials, as confirmed by our FEM analysis. A thermal shield is added, with a sufficiently deep longitudinal cut to allow for flux penetration, and the assembly is fixed onto a supporting metal plate (the "back plate") to allow thermal and electrical contact with the vacuum vessel, as shown in Fig. 2b. Finally, the coil



Fig. 3. Schematic sketch of the finite element model used for the ITER HF pickup coil: The actual meshing for the various components was calculated with ANSYS Workbench-Solid 117. The inset shows the simple one-turn winding assembly used for the thermal loads analysis; the six individual combs in the ITER original design have been modeled as one single component for each turn of the winding pack and are indicated by the thicker sections in the ceramic body. 
is electrically connected to the in-vessel wiring via an ad-hoc platform.

\section{FINITE ELEMENT ANALYSIS OF THE ITER DESIGN}

To efficiently perform the FEM analysis, and although the overall dimensions of the original design sensor were retained, a number of geometrical simplifications were made to achieve a correct meshing. First, the wiring has been considered as a single independent volume (hexagonal prism with a wall thickness equivalent to the wiring diameter and mean radius equivalent to the winding radius). This represents the worst-case scenario if (some of) the wires touch each other, because the current density would not be confined in the wire but transverse current propagation would exist. The conductance of this single sheet was set equal to the sum of the conductances of the individual winding loops. Second, and again to allow a better meshing of the assembly, the $0.5-\mathrm{mm}$ copper felt foreseen behind the back plate was not considered in the analysis. Third, the six individual combs making up the guiding grooves for one turn were modeled as one single component. Fourth, a thin shield was carved in the back plate to provide a smooth mesh transition in the region between the shield and the thicker back plate. Figure 3 shows the solid element model used for this analysis, calculated with ANSYS WorkbenchSolid 117. ${ }^{\mathrm{f}}$ More details on the geometrical simplifications used for this solid element model, on the applied load constraints, and on the overall results of this FEM analysis can be found in Ref. $15 .^{\mathrm{g}}$

For the applied loads, we have considered a constant toroidal field $B_{z}=10 \mathrm{~T}$ and a poloidal field change $d B_{y} / d t=200 \mathrm{~T} / \mathrm{s}$, as given by the current ITER load specifications. ${ }^{16 \mathrm{~h}}$ We have aligned the coil longitudinal axis to this $z$-axis, since this represents the worst-case load scenario. ${ }^{15 \mathrm{~g}}$ Figure 4 shows the test waveforms. The poloidal field component $B_{y}$ is rapidly ramped from zero to a maximum value, where it then remains constant. The toroidal field component $B_{z}$ also remains essentially constant $\left(B_{z} \sim 10 \mathrm{~T}\right)$, and the third $B$-field component $\left(B_{x}\right)$ was considered to be negligible $\left(B_{x} \sim 0\right)$. Although this is clearly just one among many possible cases of disruptioninduced magnetic fields in ITER, it was felt that this particular orientation gives a good modeling and understanding of the worst-case scenarios for components located at the current nominal position of the HF magnetic

\footnotetext{
${ }^{\mathrm{f}}$ For details on the ANSYS software code, see http://www. ansys.com (current as of Oct. 27, 2010).

${ }^{g}$ All documents in Ref. 15 are available by request to duccio. testa@epfl.ch.

${ }^{\mathrm{h}}$ The document in Ref. 16 is available by request to duccio. testa@epfl.ch.
}

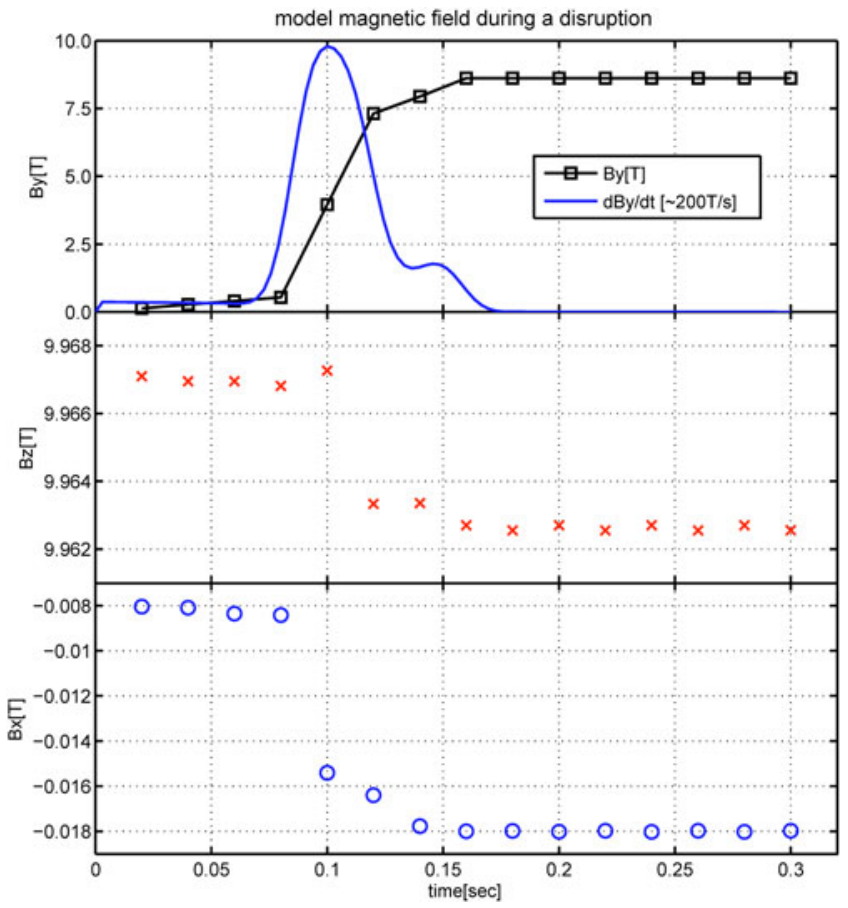

Fig. 4. The evolution of the poloidal magnetic field $B_{y}$ versus time during a disruption with $\max \left(d B_{y} / d t\right) \sim 200 \mathrm{~T} / \mathrm{s}$ between $t=0.07 \mathrm{~s}$ and $t=0.12 \mathrm{~s}$; the toroidal field component $B_{z}$ remains essentially constant, and the third $B$-field component $\left(B_{x}\right)$ has been set to be negligible.

sensors; this approach is also consistent with the work reported in Ref. 17.

For imposing the constant toroidal field, we have used the ANSYS vector potential formulation on an infinite air box that surrounds the model: The edge flux can be used to implement the external magnetic field. However, the same formulation cannot be superimposed and applied simultaneously along the other axes. To describe the poloidal field variation, we used the common experimental test-bed model approach, which includes a Helmholtz coil assembly. In this case, a time-varying current applied to the Helmholtz assembly produces the required time-dependent "disruption" poloidal magnetic field, which can then be superimposed to the previously imposed toroidal field.

This Helmholtz assembly includes two $N$-turn coils of radius $R=0.4 \mathrm{~m}$ separated by a distance equal to their radius, carrying the same electrical current $I_{H E L M}$ in the same direction. The magnetic field induced in a region extending between the centers of the assembly out to approximately one-fifth of their diameter has a nearly uniform spatial distribution, with the value in the center approximated as $B(\mathrm{~T})=(4 / 5)^{3 / 2} \mu_{0} N I_{H E L M} / R=5.62 \times 10^{-9} \mathrm{~T}$.

\footnotetext{
${ }^{\mathrm{i}}$ The document in Ref. 17 is available by request to duccio.
} testa@epfl.ch. 


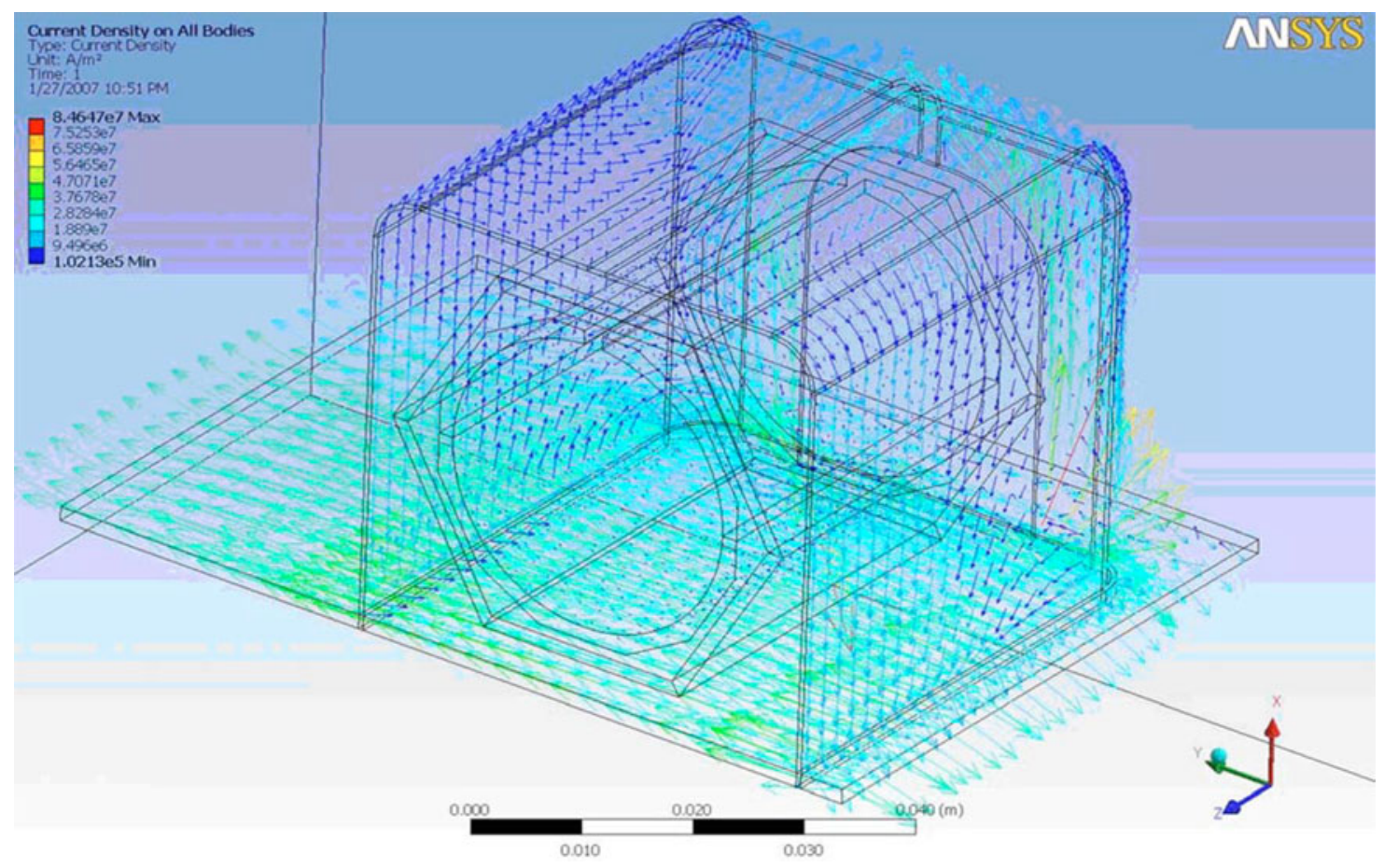

Fig. 5. Distribution of the eddy current density in different parts of the HF pickup coil assembly during the disruption time interval (i.e., at the maximum of the $d B_{y} / d t$ variation, which occurs between $t=0.07 \mathrm{~s}$ and $t=0.12 \mathrm{~s}$, as shown in Fig. 4). We have zoomed at half-size of the coil for clarity of illustration. The coil longitudinal axis has been aligned with the toroidal $z$-axis as this represents the worst-case load scenario.

Here, $\mu_{0}=4 \pi \times 10^{-7} \mathrm{~T} \cdot \mathrm{m} / \mathrm{A}$ is the vacuum permeability constant. To check if the time-dependent poloidal $B_{y}$ magnetic field is applied correctly, we compared the $n u$ merical results obtained with two commercial codes (ANSYS and COMSOL, ${ }^{j}$ using different meshing) and a two-dimensional (2-D) model data set computed using MATLAB directly solving the Biot-Savart law for a circular current loop. This analysis shows that the numerical (i.e., using ANSYS and COMSOL) and test (i.e., solving directly the Biot-Savart law) values of the magnetic field are within 3\%, the model results being slightly lower than the numerical ones. The FEM has shown that the numerical calculation of the magnetic field using ANSYS and COMSOL can be subject to variations of approximately $\pm 10 \%$ depending on the different approaches for the meshing, such as the mesh density and the choices for the mesh elements. The COMSOL results are very uniform and typically $10 \%$ below those in the model, whereas the ANSYS results are generally within $5 \%$ of the model, although the scatter is larger because of the different flexible meshing approaches used for this analysis.

In our FEM calculations, we used the following boundary conditions:

\footnotetext{
${ }^{\mathrm{j}}$ For details on the COMSOL software package, see http:// www.comsol.com/products/ (current as of Oct. 27, 2010).
}

1. Magnetic flux parallel boundary conditions are applied to all external faces of the air box, therefore forcing the field to be parallel to the boundary.

2. All edges within the boundary plane are defined to have a zero vector potential.

3. The whole HF pickup coil and the surrounding air box are defined as conductors, with $V=0$ on one of the faces of the surrounding air box.

Unless specifically stated otherwise in Secs. III.A through III.D, the actual materials foreseen for the ITER HF magnetic sensors have been used in our FEM analysis, namely, tungsten and copper for the wire, SS-316 for the metallic core and the support plate, and alumina for the ceramic body. For all their properties, we used the ITER Material Properties Handbook. ${ }^{18 \mathrm{k}}$

\section{III.A. Electromagnetic Force Analysis}

In this analysis we assume that the shield, the support plate, and the coil core are made of SS-316, with the winding made of tungsten, as in the ITER design. To obtain the electromagnetic forces and torques (computed

\footnotetext{
${ }^{\mathrm{k}}$ The document in Ref. 18 is available by request to duccio. testa@epfl.ch.
} 
in each element from $F=j \times B$ ), the current density and the magnetic field were first computed. The time profile shows that the eddy currents increase during the disruption (from $\sim 0.08$ to $\sim 0.12 \mathrm{~s}$ ), and then they reduce to zero when $d B_{y} / d t$ decreases to zero: From that moment the assembly is only subject to the static magnetic field. The spatial distribution of the eddy current density in the entire assembly during the disruption is shown in Fig. 5. The calculation was performed using a SS-316 core and a thermal shield with a longitudinal cut, to break up circulating eddy currents in the coil assembly. We note that no spots with a very high eddy current density exist with this arrangement, which proves that the whole assembly will resist the induced electromagnetic loads during the reference ITER disruption. These loads are slightly higher in the thermal shield than in the pickup coil itself, and the maximum eddy current load appears at the points where the thermal shield attaches to the supporting back plate and on the back plate itself.

Figure 6 shows the time evolution of the value of the eddy current density $J_{T}$ spatially averaged separately over different elements of the assembly: on the supporting back plate (Fig. 6a), on the thermal shield (Fig. 6b), on the winding pack (Fig. 6c), and on the bobbin (Fig. 6d). Having calculated the eddy current density $J_{T}$ and the local magnetic field $B$ via this transient magnetic analysis, we then computed the resulting $J_{T} \times B$ forces and applied them as a load for the structural analysis. The total electromagnetic peak force is expected to be $\sim 20 \mathrm{~N}$ and the total peak torque to be $\sim 41 \mathrm{~N} \cdot \mathrm{m}$. This analysis clearly demonstrates that, when using the actual material foreseen for this magnetic sensor, and given the geometrical simplifications mentioned when modeling its shape, the loads induced by the eddy currents are all within the structural limit of the assembly for this particular orientation of the disruption-induced magnetic field, which was taken to be the worst-case scenario foreseen in ITER.

\section{III.B. Coupled Electromagnetic and Structural Analysis}

The forces computed by the electromagnetic analysis were then used as the input static loads for the calculation of the displacements and stress distribution in the



(a)

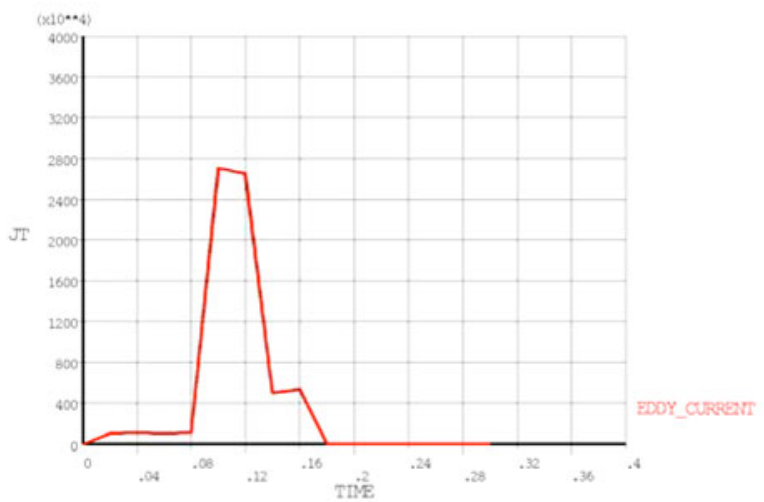

(c)

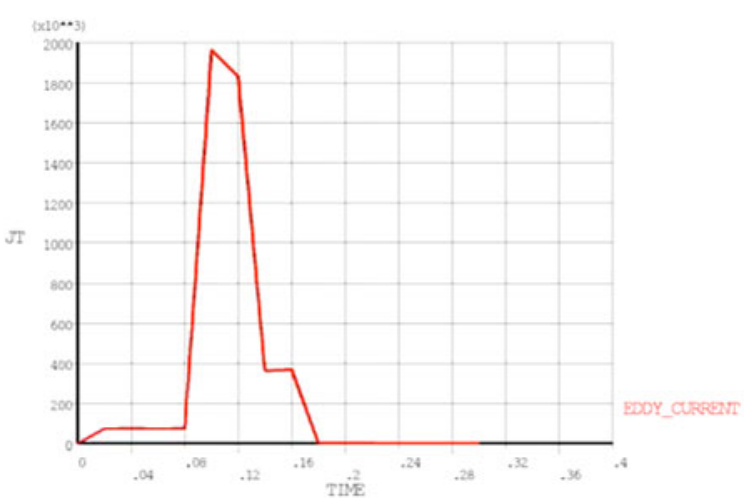

(b)

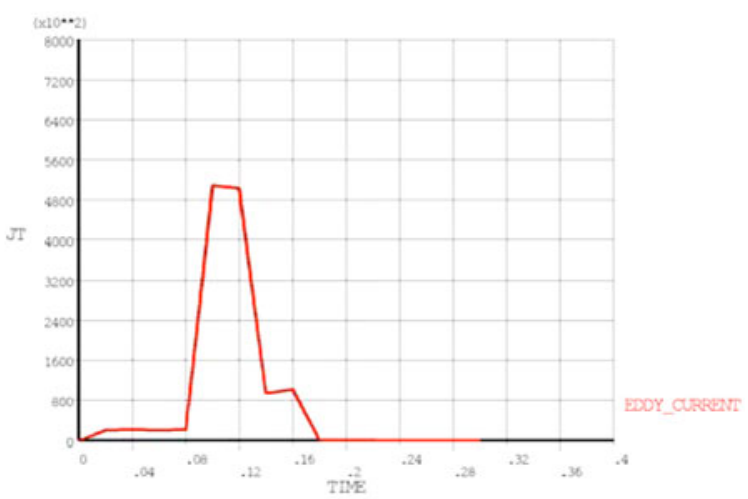

(d)

Fig. 6. Spatially averaged profile versus time of the eddy current density $J_{T}\left(\mathrm{~A} / \mathrm{m}^{2}\right)$ on different parts of the assembly, with the peak value of $J_{T}$ reached at $t \sim 0.1 \mathrm{~s}$, on (a) the supporting back plate (max $\left.J_{T} \sim 4.2 \times 10^{5} \mathrm{~A} / \mathrm{m}^{2}\right)$, (b) the thermal shield $\left(\max J_{T} \sim 1.95 \times 10^{6} \mathrm{~A} / \mathrm{m}^{2}\right)$, (c) the winding pack $\left(\max J_{T} \sim 2.75 \times 10^{7} \mathrm{~A} / \mathrm{m}^{2}\right)$, and $(\mathrm{d})$ the bobbin $\left(\max J_{T} \sim 4.9 \times 10^{5}\right.$ $\left.\mathrm{A} / \mathrm{m}^{2}\right)$. 
HF pickup coil. The assembly as a whole is supported in 12 different places, 4 of which are on the back of the support plate, i.e., the spot welds of the coil casing to the vacuum vessel. To perform this analysis, the bobbin and the winding pack were kept at fixed positions to simulate electrically insulated supports. The resulting equivalent stresses and deformation were calculated at the time of the maximum in the eddy current distribution, between $t=100 \mathrm{~ms}$ and $t=120 \mathrm{~ms}$. The Von Mises equivalent stresses in various components of the assembly during the disruption are shown in Fig. 7. Although some peak stresses reach a very high value, $\sim 100 \mathrm{MPa}$, particularly around the various attachment points, the average values are within an acceptable range of 10 to $25 \mathrm{MPa}$.

\section{III.C. Thermal Analysis}

Because of the different thermal properties of the materials used in the assembly of the HF pickup coil, it is important to understand how the winding pack will react when the assembly is subject to thermal loads. Basically, either the size of the supporting body (the bobbin and the ceramic combs mounted on it) increases more than the total length of the wire, so that tension will be generated in the winding pack itself, or the length of the wire increases more than the size of the supporting insulating former, in which case only the preload self-tension is added. In the first case, we have to take care that the elastic limit of the wire material is not exceeded, while in the second, we have to verify that the overall geometry of the wiring does not become "loose," when some parts of the winding pack might escape from the guiding grooves. As the HF pickup coil is made of two identical layers of 33 identical turns each, we considered one winding and the corresponding portion of the ceramic body (with the six individual combs again modeled as one component, as shown in the inset in Fig. 3) to simplify the thermal load analysis, still keeping its generalities.


Fig. 7. The equivalent Von Mises stress calculated in various components of the pickup coil assembly at the maximum in the eddy current distribution, between $t=100 \mathrm{~ms}$ and $t=120 \mathrm{~ms}$, in (a) the thermal shield, (b) the support plate, (c) the winding pack, and (d) the bobbin. 
Two specific reference cases were considered for this analysis, with different material properties, $\alpha$ being the CTE for the selected material.

\begin{tabular}{|l|l|l|}
\hline & \multicolumn{1}{|c|}{ Wire Material } & Coil Bobbin and/or Body Material \\
\hline Case 1 & $\begin{array}{l}\text { Copper, } \\
\alpha_{\mathrm{Cu}}=16.5 \times 10^{-6}(1 / \mathrm{K})\end{array}$ & $\begin{array}{l}\text { Ceramic body: } \\
\text { alumina, } \alpha_{\mathrm{CER}}=1 \times 10^{-6}(1 / \mathrm{K})\end{array}$ \\
\hline Case 2 & $\begin{array}{l}\text { Tungsten, } \\
\alpha_{\mathrm{W}}=4.5 \times 10^{-6}(1 / \mathrm{K})\end{array}$ & $\begin{array}{l}\text { Metallic bobbin: } \\
\text { SS-316, } \alpha_{S S}=12 \times 10^{-6}(1 / \mathrm{K})\end{array}$ \\
\hline
\end{tabular}

First, we consider the case where the mechanical tensions in the wire and in the body are not critical, which is obtained by using a copper wire and an alumina body. In this case it is not important whether or not there is a metallic bobbin; hence, this element is not considered in this analysis. The length of the wire will increase more than the circumference of the body, as $\alpha_{\mathrm{Cu}}=16.5 \times 10^{-6}$ $(1 / \mathrm{K})$ compared to $\alpha_{C E R}=1 \times 10^{-6}(1 / \mathrm{K})$. In this situation we have to ensure that there will still be tension in the wire after its increase in length, so as to keep the correct shape of the winding. This can be achieved by applying a sufficiently high initial preload.

Second, we consider the case producing the biggest mechanical tension between the wire and the body (through compression of the ceramic spacer). This is obtained using a SS-316 bobbin and a tungsten wire, because the bobbin will be subject to a large increase in size, as $\alpha_{S S}=12 \times 10^{-6}(1 / \mathrm{K})$ compared to $\alpha_{\mathrm{W}}=4.5 \times$ $10^{-6}(1 / \mathrm{K})$. Now, we have to ensure that the wire will survive the mechanical tension induced by such expansion. As a number of simplifications in the actual design of the pickup coil, and specifically for the winding pack, had to be taken to perform the FEM analysis efficiently, we verified the numerical calculations of the thermal loading in these two cases using simplified analytic modeling. The results of these two approaches are in good general agreement, supporting the conclusions of our thermal analysis.

For our FEM and analytical calculations, we considered the nominal temperature increase in the coil assembly to be $\Delta T=30^{\circ} \mathrm{C}$, in line with the current estimate for the length of one ITER pulse ${ }^{16}$. This is a lower bound on the maximum temperature increase to which the ITER vacuum vessel might be subjected during cyclic plasma operation, namely, up to $\Delta T=150^{\circ} \mathrm{C}$ as an upper bound. Baking at $T>150^{\circ} \mathrm{C}$ for much longer thermal cycles is not considered as an operational concern. It is clear that if problems are already found with the HF sensor design using this lower temperature increase, then these can only become much worse for $\Delta T=150^{\circ} \mathrm{C}$. For the calculations, no initial preload or stress in the wire is assumed at the initial ambient temperature of $25^{\circ} \mathrm{C}$, which was chosen to simulate the initial conditions after manufacturing and installation but before the first baking cycle of the ITER vacuum vessel.
For the first simulation, which uses a ceramic body, the CTE of the ceramic body is much lower than that of the wire. Therefore, no tension is added by the body to the wire, and the only real issue is to control whether the deformation of the wire itself, when subject to the nominal temperature increase $\Delta T=30^{\circ} \mathrm{C}$, allows the wire to remain captive inside the guiding grooves. At the final temperature of $55^{\circ} \mathrm{C}$, the maximum deformation of the ceramic body (i.e., of the length of one turn around the grooving) is calculated to be $\sim 12.6 \mu \mathrm{m}$ (using the FEM analysis, compared to $\sim 8 \mu \mathrm{m}$ analytically), whereas the one-turn length of the copper wire increases by $\sim 63 \mu \mathrm{m}$ (both analytically and FEM). This differential thermal expansion between one turn of the wire and one turn of the ceramic body reduces the tension in the copper wire due to dilatation, which can be calculated in the FEM analysis or analytically using Hooke's law, to give $\sigma_{D I L} \approx$ $56 \mathrm{MPa}$. To keep the correct shape of the winding pack, an initial preload tension of $\sigma_{P R E-L O A D}=\sigma_{D I L} \approx 56 \mathrm{MPa}$ needs to be applied, which is very close to the ultimate tensile strength of copper $(70 \mathrm{MPa})$ and requires $\sim 16$ $\mathrm{N} /$ turn to be applied during the winding operation.

For the second simulation, which uses a SS-316 bobbin and a tungsten wire guided through individual ceramic spacers to simulate the worst case of traction in the wire and compression in the body, there is a tension in the wire generated by the differential thermal expansion with the bobbin. The initial length of one turn of the wire (and bobbin) is $L_{0}=127.5 \mathrm{~mm}$, increasing to $L_{\text {WIRE }}=$ $127.517 \mathrm{~mm}$ and $L_{\text {BOBBIN }}=127.546 \mathrm{~mm}$, respectively, when subject to a temperature increase of $\Delta T=30^{\circ} \mathrm{C}$. At the final temperature of $55^{\circ} \mathrm{C}$, the equivalent tension in the wire reaches a value of the order of $160 \mathrm{MPa}$ (calculated analytically, compared to $\sim 150$ MPausing FEManalysis), which includes the constraint due to the initial wiring preload applied during the winding process. As the ultimate strength of the tungsten wire is $\sim 600 \mathrm{MPa}$, this situation is acceptable from a static point of view; i.e., the wire will not break. Such constraints can become critical if a ceramic grooving with thin walls is used to separate each winding turn from the adjacent ones, particularly when considering that these stresses could be repetitive, such as during a series of disruptions.

\section{III.D. Conclusions from the Electromagnetic, Structural, and Thermal Analysis}

The Helmholtz coil modeling setup has been extensively used for a numerical validation of the simulations run using ANSYS and COMSOL, and the FEM approach presented here allows good reproducibility of the results. The limits of the method are the required computational time and the difficulty of meshing three-dimensional (3-D) wires: Thus, a "worst-case" approach was analyzed, which assumed a continuous cylinder-like wiring and one particular orientation of the coil's longitudinal axis and the disruption-induced poloidal magnetic field. Within this 
framework, and using the actual materials foreseen for the ITER HF magnetic sensor, our analysis indicates that the maximum stress in the tungsten winding is $\sim 63 \mathrm{MPa}$, compared with an ultimate strength of $550 \mathrm{MPa}$, and 98 $\mathrm{MPa}$ in the thermal shield. Without considering the peak values, the maximum Von Mises stress is within the range of 20 to $50 \mathrm{MPa}$ in the tungsten wire and 10 to $25 \mathrm{MPa}$ in the thermal shield. These values are of no concern for a metallic support, but further analysis would be required should a ceramic material be used for the coil core instead of the currently intended SS-316.

Because of the difference in the thermal expansion between the SS-316 bobbin, the ceramic body acting as insulating spacers for the winding pack, and the wire itself, heating in the HF pickup coil assembly produces displacements and stresses in the wire. Depending on the initial preload applied to the wire, our analysis suggests that this effect could break the wire or the thin edges in the ceramic spacers even when considering a very moderate temperature increase of $\Delta T=30^{\circ} \mathrm{C}$. Although these thermal analyses were performed in a static environment, it is clear that the same considerations apply for the dynamical loads associated with the foreseeable cycles in temperature fluctuations, which are further compounded by the added risk of mechanical fatigue.

Hence, our FEM and analytical analysis has identified the differential thermal expansion between the ceramic spacers and the winding pack as the main potential risk for the mechanical integrity of the Mirnov-type HF magnetic sensor assembly. The analysis of this potential problem has therefore provided the essential focus for our prototyping program, which was tackled by designing insulating formers with different guiding grooves for the wire and using different materials for the wire itself.

\section{PROTOTYPE VARIANTS OF THE ITER DESIGN AND THE RP TECHNOLOGY}

The ITER design for the Mirnov-type coil, shown in Fig. 8, uses straight (i.e., perpendicular to the coil's longitudinal $z$-axis) guiding grooves with very thin edges, which suggests that the corners of the grooves may be easily breakable because of the unavoidable tension (and movements) in the winding pack. There are six individual guiding grooves (i.e., the combs) for each hexagonal turn of the winding, and these grooves are not aligned on one single $x-y$ plane. Therefore, with this design the helicoidal winding path is separated into straight sections (running along one short portion of one $x-y$ plane perpendicular to the coil's $z$-axis) and oblique sections (connecting one portion of the $x-y$ plane to the next portion, which is now in an $x-y$ plane slightly displaced along the $z$-axis): This gives rise to tight angles in the wire at the corners of the spacers, which further compounds the weakness of the thin edges in the insulating former. This was

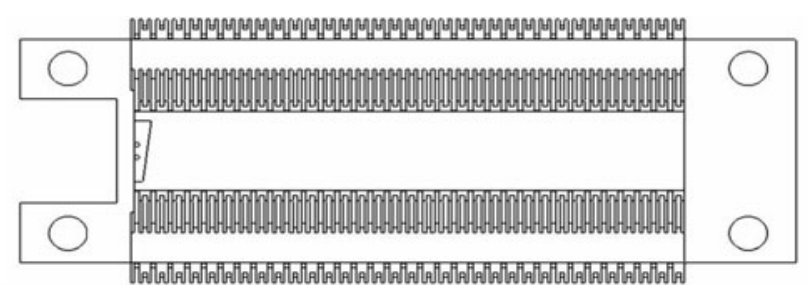

(a)

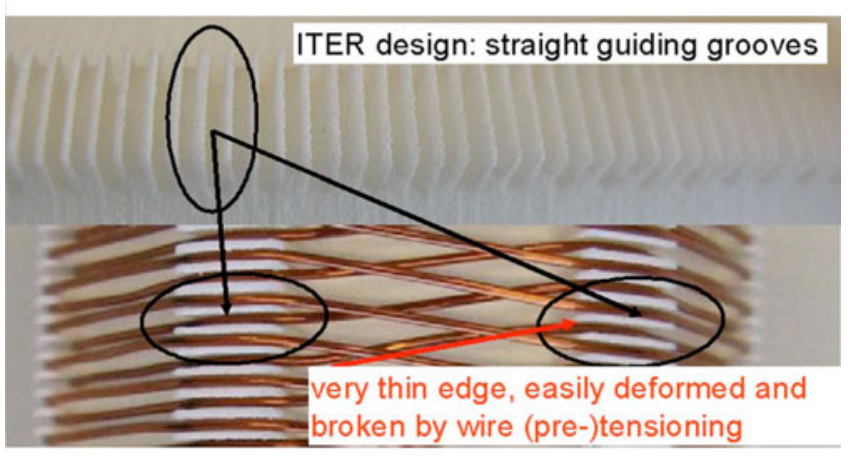

(b)

Fig. 8. The ITER design of the HF Mirnov-type pickup coil: This design uses straight and very thin guiding grooves, which implies that the corners of the grooves are easily breakable during the winding process (and during operation when installed in-vessel) because of the unavoidable tension in the winding pack. In the bottom frame (b), a portion of the insulating former showing the straight guiding grooves and one of the as-built prototypes using a copper wire are shown. With this design, the helicoidal winding path is separated into straight (i.e., perpendicular to the coil axis) and oblique sections, giving rise to tight angles in the wire at the corners of the spacers.

assessed as being an unsatisfactory assembly process for the winding pack and as presenting a major risk during the manufacturing, assembly, and operation of the coils, as there is a phase when the winding pack assembly (wire and combs) is still loose on the supporting bobbin. To ensure that the wire and the grooves are kept in good mechanical condition during the manufacturing and assembly process, and that the winding is less prone to nonuniform displacements between its various turns during operation, it is far preferable to have a helicoidal winding path with the same pitch along the whole length of the coil, i.e., one which is not separated into straight and oblique sections. Hence, two further modified designs for the insulating former were prototyped.

The "first modified version," shown in Fig. 9, has slanted, but still straight, grooves. These are cut at an angle with respect to the coil's longitudinal $z$-axis, and there is also a smooth angle in the groove itself, which guides the wire along its hexagonal path in the $x-y$ plane. The principal advantage of this design is that the wire is more uniformly guided, so that the helicoidal winding 


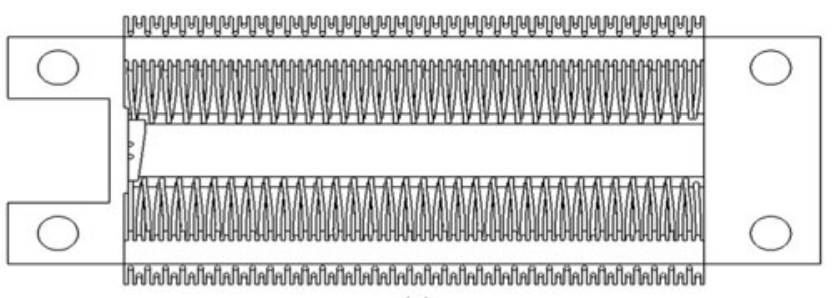

(a)

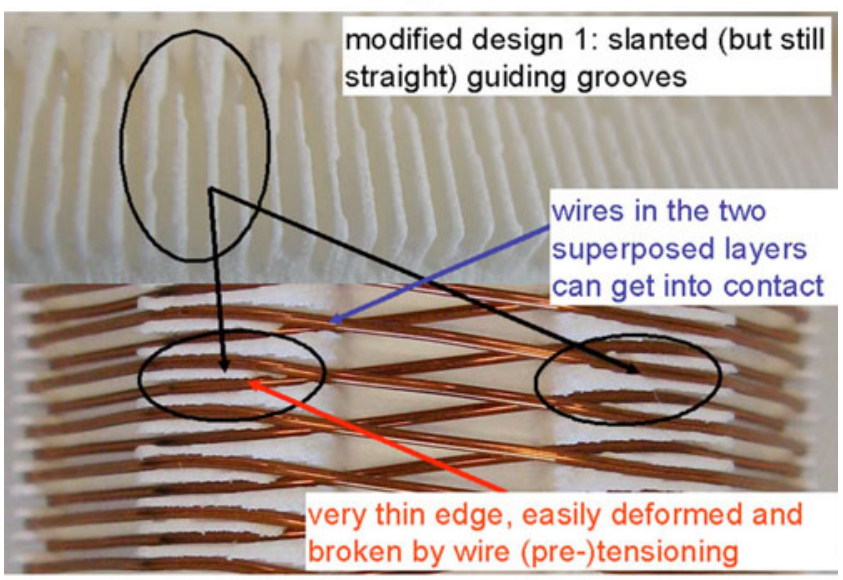

(b)

Fig. 9. The first modified prototype version, with slanted but still straight guiding grooves: There are still thin edges in the insulating former, and now there is also the risk of the wires in the first and second layer coming into contact. In the bottom frame (b), a portion of the insulating former showing a detailed view of the slanted guiding grooves and one of the as-built prototypes using a copper wire are shown.

path has a more constant pitch along the coil's length: Now tighter angles in the wire can only occur in the sections where the wire goes through a groove, not at its corners, which significantly reduces the stresses in the thin edges caused by the (pre-)tensioning and/or movement of the wire.

In the "second modified version," shown in Fig. 10, passing holes have been added to the guiding grooves, so that the top and bottom layers are fully separated and cannot contact each other. With this design we then have a helicoidal winding path with a very uniform pitch along the coil's length, but to achieve this, the passing holes (and the grooves as well, although to a smaller degree) must be curved in both the $x$ and $y$ directions (i.e., they have a 2-D curvature radius).

These prototypes were manufactured using the socalled RP technology to make the insulating former instead of the ceramic material envisaged in the ITER original design for the HF pickup coil. A recent example of scientific application of the RP technology is given in Ref. 19. A simple description of the RP technology can



(a)

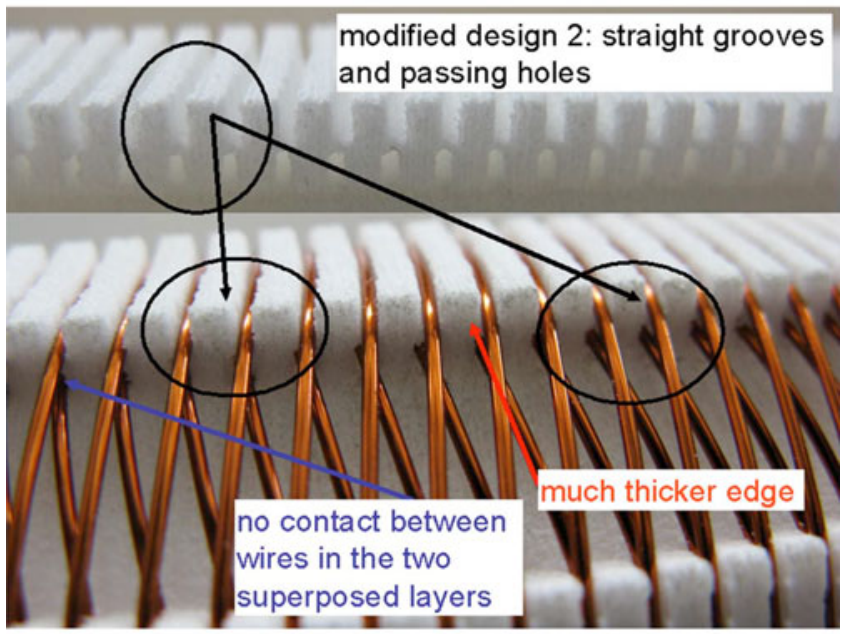

(b)

Fig. 10. The second modified prototype version, with 2-D, curved grooves and passing holes; now the wires' in the first and second layer coming into contact is avoided as each wire passes through separate holes in the grooving of the insulating former, and the edges in the insulating former are much thicker. In the bottom frame (b), a portion of the insulating former showing a detailed view of the straight guiding grooves and passing holes and one of the as-built prototypes using a copper wire are shown. With this design we also have a helicoidal winding path with a very uniform pitch along the coil's length.

be found on the Internet site of the manufacturer we selected": "Laser Sintering is a rapid prototyping process of fusing together layers of powder into a 3D model by a computer-directed heat laser. 3D CAD data of a new product or prototype component is sliced into layers, and the lasers sinter (melt) the powder layer by layer. Additional powder is deposited on top of each solidified layer and again sintered."

This technology allows us to create all shapes made with a computer-aided design (CAD) program. The basic material for the RP technology is PA12, with other variants for improved thermomechanical properties. The standard PA12 was chosen for the manufacturing of the prototypes of the ITER HF pickup coils since neither

${ }^{1}$ Composites Busch SA, http://www.compositesbusch.ch/ (current as of Oct. 27,2010 ) 
thermal nor mechanical (stress and strain) tests were going to be performed on these prototypes. We recall that the principal purpose of using a polyamide body rather than a ceramic body is simply to allow winding of wires of different material to check the feasibility of this process on thin spacers, since this was found to be the sole issue for the integrity of the ITER-designed Mirnov-type magnetic sensor in our FEM analysis; the different mechanical properties of polyamide and ceramic are not expected to affect the assessment of the feasibility of the winding process. The relative dielectric constant of the PA12 polymer is $\varepsilon_{r}=3$ at $1 \mathrm{MHz}$ (compared to $\varepsilon_{r} \sim 4.5$ for $\mathrm{Al}_{2} \mathrm{O}_{3}$ and Shapal-M and $\varepsilon_{r} \sim 12$ for $\mathrm{ZrO}_{2}$ at $1 \mathrm{MHz}$ ), which impacts the electrical properties of the prototypes in a simple scalable way through the dependence of the selfcapacitance on $\varepsilon_{r}$.

Finally, two essential features of the ITER design for the HF pickup coil are the presence of the SS-316 bobbin, with or without a longitudinal cut, and of a thermal shield/cover, which always has a deep longitudinal cut, as shown in Fig. 2 and described in more detail in Sec. II. The addition of the SS-316 bobbin and thermal shield changes the HF electrical characteristics of the pickup coil because they act as a capacitively loaded one-turn secondary of a transformer, the primary being the winding pack itself. Hence, both parts were manufactured in SS-316 to perform a complete analysis of the sensor's electrical properties.

In summary, the ceramic body of the HF coil has been manufactured in one single part using the RP technology, allowing us to characterize independently (a) the assembly of the winding pack by using different grooving layout on the ceramic body and (b) the electrical characteristics of the coil with and without the insertion inside the body proper of the SS-316 bobbin. Winding tests were then performed using a copper wire and a tungsten wire on each prototype version to test the robustness of the winding process. Therefore, three different prototypes were designed for the ceramic body, and each manufactured in various examples, for each design using the RP technology, and wound with a copper wire and a tungsten wire. Some prototypes are shown in Fig. 11, namely,

1. a body with the exact shape and geometry of the ITER design, version V0 (as in Fig. 8).

2. a body with modified slanted, but still straight, grooving, version V1 (as in Fig. 9).

3. a body with modified grooving using passing holes, version V2 (as in Fig. 10).

\section{IV.A. Conventional Prototype Design: Winding Tests Using Copper Wire}

The first winding tests were performed using a copper wire, and the winding pack was assembled on each prototype to test the winding process. The prototype version V0 (ITER design) was indeed very simple to wind, but on close visual inspection there is a significant deformation in the groove walls at each turn change, as shown in Fig. 12a. This deformation is acceptable

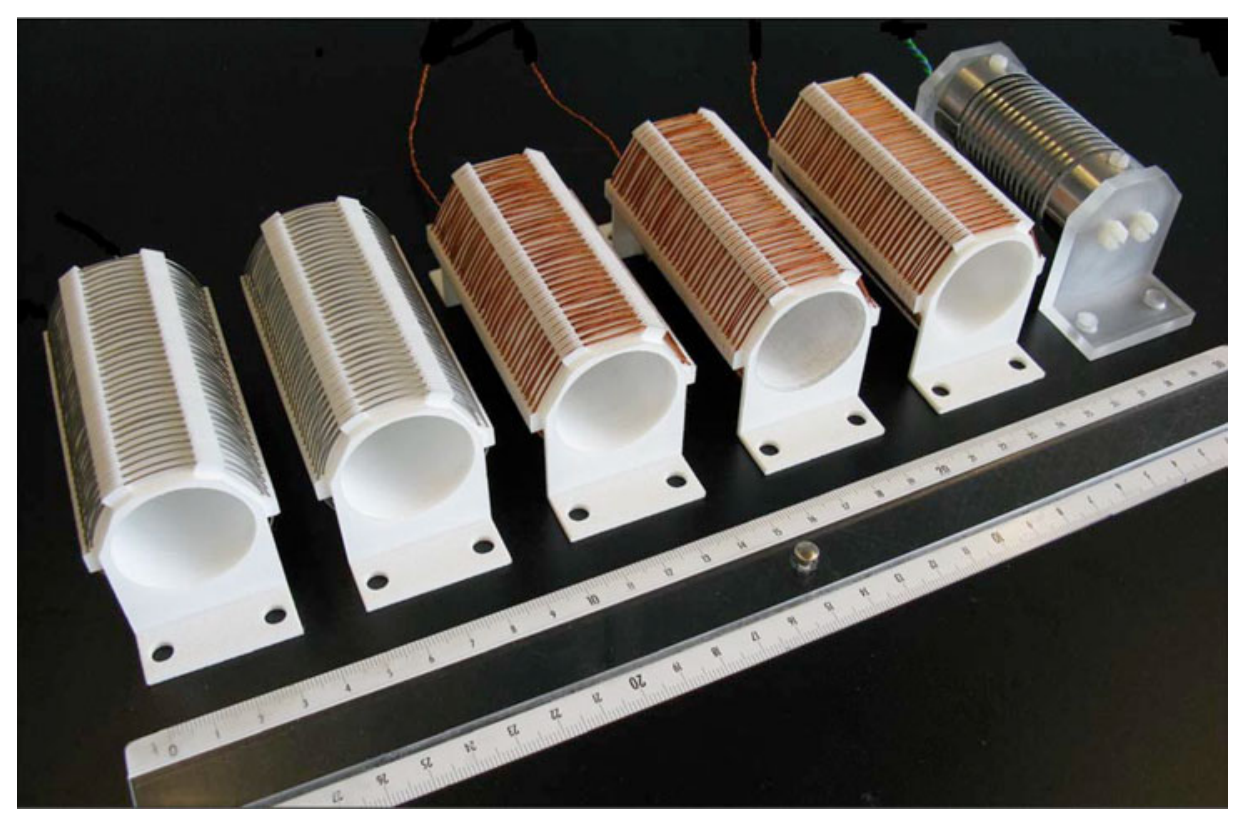

Fig. 11. From left to right, five examples of the as-built prototypes for the ITER HF pickup coil, manufactured using the RP technology, with different grooving design and wound with tungsten and copper wire; the rightmost coil in SS-316 was made using the laser-cutting technique described in Sec. IV.C, shown here as mounted on its Plexiglas support. 




(a)

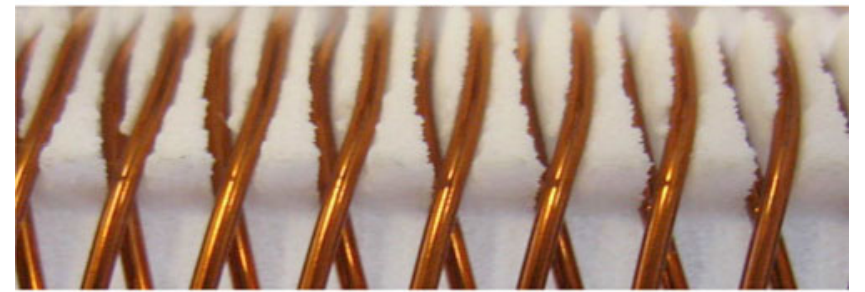

(b)

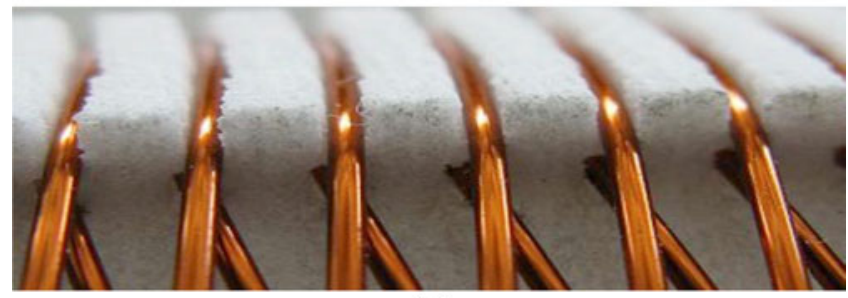

(c)

Fig. 12. (a) Detailed zoom of the straight grooving for the V0 prototype version (ITER design): Note the bending and very significant deformation in the groove walls at the top of each turn, which makes this design and assembly process for the winding pack unsuitable for use in ITER. (b) Detailed zoom of the slanted grooving in the V1 prototype version, which clearly shows the deformation in the grooving of the ceramic body around the regions where the winding in the two superposed layers cross each other. (c) Detailed zoom of the grooves and passing holes in the V2 prototype version: It is immediately clear to see that this winding pack assembly is mechanically much stronger than the other two designs, but the 2-D curvature of the passing hole makes it almost impossible to wind with copper unless the holes are preformed using a much harder wire, such as tungsten, in our case.

when using slightly deformable polyamide, but as the insulating former of the ITER coil will be made of ceramic, we conclude that there is an obvious risk of breaking the groove walls because of the brittleness of the ceramic. This was later confirmed with some semidestructive testing on thin samples in $\mathrm{Al}_{2} \mathrm{O}_{3}$, Macor, and Shapal-M. Hence, we suggest that this particular design combination (copper wire, straight guiding grooves in the ceramic body) should not be considered for a conventional Mirnov-type HF pickup coil to be used in ITER unless much thicker edges are allowed in the ceramic body - at the expense, however, of a reduced number of turns and, hence, effective area.
The prototype version V1 was also very simple to wind. The wire guide between the two slanted guiding grooves guarantees a good assembly process for the winding pack. However, there is the same or an even worse problem as for version V0: The tension in the wire between the first and the second layer creates a narrow edge in the grooves, and the wire is guided through by this edge. This narrow edge can be easily seen in the zoomed-in Fig. 12b: The PA12 is clearly deformed in the region where the two superposed layers cross each other. Furthermore, we have observed that with this design it is quite possible that the wires in the two superposed layers may contact, as these two layers are very close to each other in certain sections of their helicoidal paths. This might occur either during assembly because of wire pretensioning, or more likely during operation because of wire displacement. This would effectively short out a section of the winding pack, hence completely changing the sensor's electrical properties and effective area. Such a "collision" between the two layers also increases the brittleness of the groove edges. This edge is very unlikely to be of sufficient strength when using any of the ceramic materials that are required for the engineering design of the ITER Mirnov-type pickup coil. Even if this first modified design for the insulating former has a better winding appearance than the ITER design, this solution is still not considered to be adequate.

The prototype version V2 was found to be almost impossible to wind with the copper wire as the passing holes are very long, have a very small diameter, and are curved in two directions. Hence, passing through the holes becomes very difficult with the copper wire, which is very ductile. The winding was only possible by preforming the passing holes using a harder and smaller wire such as tungsten. As shown in Fig. 12c, the advantage of this solution clearly resides in the strength of the walls in the grooves, as their thickness is much larger than that in the $\mathrm{V} 0$ and $\mathrm{V} 1$ designs. The wire is still guided along its helicoidal path, and the two layers cannot touch now as the wires have to pass inside holes when they are closer, thus fully separating the first layer from the second layer. The walls and edges of the grooves are therefore much stronger, but the wire is more difficult to wind.

\section{IV.B. Conventional Prototype Design: Winding Tests Using a Tungsten Wire}

With tungsten wire, it was far more difficult to assemble the winding pack, as tungsten is more rigid and less ductile than copper, hence easily breakable during the winding process. Moreover, tungsten cannot be deformed easily: We obtained an almost circular shape for the winding pack, instead of the intended hexagonal one, which is furthermore skewed along the longitudinal $z$-axis of the coil (i.e., each turn does not sit on a single $x-y$ plane). Therefore, all turns effectively enclose a slightly different area, which is moreover not as well aligned 
perpendicularly to the longitudinal $z$-axis of the coil as when using a copper wire. This can have a serious impact on the reproducibility of the electrical properties of the pickup coils when considering the required series production for the ITER HF magnetic sensors and might even open the possibility of variations of effective area by mechanical shocks during operation.

The prototype version V0 was a little bit harder to wind with tungsten wire than with copper wire. The wire broke twice during the twisting phase, performed at the end of the winding assembly, but for one test coil. Furthermore, we had the same problems we experienced with the copper wire for this design, namely, the appearance of a significant deformation in the groove walls at each change of turns. No winding problems, such as breakages of the tungsten wire, appeared when testing the prototype version $\mathrm{V} 1$, but, as it essentially presents very similar problems as those seen using a copper wire, this design does not give any improvements over the ITER design. For the prototype version V2, it was, as to be expected, much easier to make the wire go through the passing holes because tungsten wire is more rigid than copper. Remember that preforming the passing holes was required when testing this design with the copper wire. However, the much larger number of handling operations required to wind this coil to get through all the passing holes is not compatible with the very low ductility of tungsten, which indeed regularly broke during the winding process. Hence, the winding pack could not be reliably assembled for this design when using the tungsten wire, and further assembly and electrical tests were not performed.

\section{IV.C. Alternative, Nonconventional Design Using Laser Cutting of a Hollow Coaxial Tube}

Based on the winding tests with copper and tungsten, it is clear that further prototyping should be performed to explore improved manufacturing and assembly solutions for the winding pack for a Mirnov-type pickup coil of conventional design. To avoid the problem of the brittleness of the ceramic body, the best solution is obtained by guiding the wire over all its length, i.e., along all the turns on each layer and across the different layers. In this case, we need to avoid small guiding grooves on the ceramic body, which would inherently lead to thin walls in the grooving.

Ideally, the mechanical and electrical requirements for the ITER HF pickup coil can be satisfied using a single metallic part with exactly the same shape of the wire in the conventional design. This would then completely avoid the winding process and all its difficulties, including the geometrical defects related to a nonperfect reproducibility of the winding. The manufacturing of a complicated ceramic coil body can then also be avoided. Moreover, a metallic winding in one single part would be much stronger than a thin wire, and the connections would be easier and stronger too. There are three possibilities for manufacturing such metallic winding in one part using standard CAD processes: (a) conventional manufacturing, (b) powder technology, and (c) laser-cutting technology.

Using conventional processes to produce a continuous metallic winding appears very challenging, as one would need a very thin wire, with a diameter ranging from $\sim 0.6$ to $\sim 1 \mathrm{~mm}$. This clearly implies a complicated manufacturing process that is likely to be almost impossible to be scaled to the production of a large number of individual components. The powder technology is expensive, essentially because it is a manufacturing method that is still in its development phase, and the resulting semiporous structure causes a clearly perceived risk of trapped volume and outgassing. The main advantage of this technology is the possibility of manufacturing very quickly, simply, and cheaply almost every imaginable shape. The laser-cutting technology is a rather inexpensive manufacturing process, and thin shapes can easily be obtained because of the diameter of the laser beam; prototypes can be easily and very cheaply manufactured. Moreover, once the manufacturing process has been defined and validated, it becomes very easy to perform a mass production with a high level of reliability and reproducibility using conventional CAD processes.

Comparing the advantages and disadvantages of each manufacturing process, we considered that the most appropriate method for our following prototyping tests was the laser-cutting technology. Our goal was to manufacture an equivalent winding pack by laser cutting a hollow metallic tube in SS-316. Industry was contacted to perform these manufacturing tests, and this resulted in the design of a test prototype, which had only one winding layer and 16 turns, hence a far smaller effective area than the $\mathrm{V} 0, \mathrm{~V} 1$, and $\mathrm{V} 2$ prototypes and than the original ITER design. This prototype is shown in Fig. 13 as mounted onto a Plexiglas support.

In this final design, the two ends of the coil are large and strong to allow for the fixing of the coil onto a support structure and an easy electrical connection. Two longitudinal cuts are then added, one at each end of the coil, to avoid creating two additional and very thick turns in the coil, which would completely change its electrical properties. For the manufacturing of the coil, a SS-316 tube was used with a length equal to that required for the finished coil without the $1.5-\mathrm{mm}$ gap between each turn, so that the total length of the tube before the laser-cutting operation is $44 \mathrm{~mm}$. With this method, the laser beam cuts across the tube along one direction, instead of having to cut a round trip over the entire length of the tube, which would have been a much more complex sequence of operations. When the laser cutting is finished, the coil can be deformed elastically to the required shape and length by forcing a gap between the turns.

This specific manufacturing process was selected essentially because of the small dimensions of the coil. In 




Fig. 13. The laser-cut prototype coil as mounted on its Plexiglas support structure, which was built in-house to constrain the coil to a fixed shape; note the clearly visible cuts at both ends of the coil, which break up the very large last winding.

fact, its equivalent wire thickness is very small $(1.5 \mathrm{~mm})$, meaning that the coil can be deformed very easily, which makes it much more difficult to manufacture it directly with the gap between the turns. With bigger dimensions of the equivalent wire width, the coil will be much more rigid; hence, it will not be easily deformed during the laser-cutting process, but this will impact its electrical characteristics. Although the coil prototype was manufactured without any problems during the laser-cutting process itself, on final visual inspection we found some defects:

1. The helicoidal twist between each turn is not constant.

2. The equivalent wire thickness of the last turn is smaller than the other ones.

3. The coil is not exactly straight or completely symmetrical along its longitudinal axis.

4. The coil is very ductile, essentially behaving like an unloaded spring.

It is clear from visual inspection that a support is needed to maintain the coil in its correct shape and to mitigate these defects. As the main goal of this prototyping test was to find out if it was possible at all to produce such a nonconventional laser-cut design, this support has been built for simplicity in-house using nonconducting Plexiglas, even if this has somewhat changed the electrical properties of this prototype with respect to the conventional prototypes built using PA12, because of the different relative dielectric constants of Plexiglas and PA12. This support is made with a cylindrical part whose role is to maintain the shape of the coil and give it the correct length. Two other parts are added on its sides to finally fix the assembly to the vacuum vessel wall. With this support, the geometry of the laser-cut prototype is much improved: The distance between each turn is now constant, and the coil is perfectly aligned along its longitudinal axis. The last geometrical defect (i.e., the smaller thickness of the last turn) will need to be corrected by revising the coil's design in collaboration with the manufacturer.

\section{IV.D. Summary and Conclusions on the Mechanical Testing}

Based on the results of our FEM analysis, the differential thermal expansion between the ceramic spacers and the winding pack was identified as the main risk for the mechanical integrity of the Mirnov-type HF pickup coil. As this problem is related to the specific details of the grooving itself and of the material selected for the wire, we decided to look for a prototyping solution that would allow us to manufacture and wind a large number of test coils with dimensions and shape equal to the ITER design so as to assess the winding process on a statistical basis and consider also the manufacturing tolerances. The best solution, in terms of costing and functionality, was the RP technology, which allowed making the body of the coil, including the grooving, in only one part.

Our general assessment is that the RP technology is indeed a very interesting tool, as it has proven to be very successful for our prototyping tasks. Any geometry can be created very rapidly (in our case, the typical procurement time was five working days from order to delivery), and the production costs are very low. For example, to fill the tank where the laser sintering process takes place, we ordered 28 prototypes for approximately $\$ 2000(\sim 1400 €)$, a cost of approximately $\$ 70(\sim 50 €)$ per coil body. On visual impression and during manual handling, the prototypes have excellent mechanical characteristics and are very strong even in their thinner regions (the wall thickness of the thinner grooves measures just $\sim 0.15 \mathrm{~mm}$ ). The mechanical strength of the part is also more than sufficient for the main manufacturing, winding, and electrical tests that need to be performed on these prototypes on a routine basis. This technology can clearly be used very effectively for future prototyping projects. In our view, these procurement advantages more than compensate for the unsuitability of the RP material for any thermal and outgassing tests, as the main manufacturing solutions can be explored first at a very low cost within a very short procurement time. Only the more advanced technical solutions would need to be explored using the much more expensive materials foreseen for use in ITER.

The principal problems we encountered during the manufacturing of the prototypes for the ITER HF pickup coil of conventional Mirnov-type design are the appearance of thin and easily breakable edges in the guiding grooves in the insulating former, and the winding operation itself, particularly when using tungsten, which also generates tension in the wire and causes a deformation in 
the shape of the coil. The assembly of the winding pack is a difficult operation to perform when the reliability of the winding process and the geometrical reproducibility have to be guaranteed to a high degree, as is the case in ITER, where we will have between 170 and $500 \mathrm{HF}$ magnetic sensors, depending on the chosen system implementation. ${ }^{12}$ Hence, although each sensor will be individually tested, the reproducibility and the reliability of the assembly process must be as high as possible, if nothing else simply to facilitate the data analysis and the possible swapping of faulty sensors with unused sensors.

Our test program has indicated that, when using straight (ITER design) and slanted (our first modified design) grooves, the assembly of the winding pack could be performed easily, but with a problem of brittleness due to the thickness of the groove wall (in PA12); this problem is further aggravated by the lack of guiding of the wire. This will inevitably lead to breakages, either during the manufacturing process or during operation because of (even) minor movements in the coil's assembly due to $j \times B$ forces. This was also practically verified in the JET clean room using ceramic spacers made in Shapal-M, Macor, and alumina $\left(\mathrm{Al}_{2} \mathrm{O}_{3}\right)$, with edges of similar thickness, although not with the exact shape overall as those in these prototypes.

When using a second modified design which has 2-D-curved guiding grooves together with passing holes, the insulating former becomes much stronger than the first two solutions; i.e., it does not have thin and easily breakable edges along which the wire is guided through its helicoidal path. This is in principle the most promising avenue to explore for optimizing the assembly of the winding pack for this conventional HF pickup coil, even if this design is not easily wound. Furthermore, it is also felt that such a design for the insulating former may be very difficult (or at least requiring very lengthy and expensive processes) to manufacture using conventional CAD machines with the ceramics foreseen for ITER (i.e., with required hardness, such as Shapal-M, alumina, boron oxide, or zirconium oxide), as based on our experience with these materials and confirmed by informal discussions with a number of manufacturing companies of ceramics components.

The winding tests performed using a copper wire were in general successful, apart from the need of preforming the passing holes with a harder material. The winding tests performed using tungsten wire demonstrated that that wire can easily be broken during the many handling processes required to assemble the winding pack. Therefore, this material is not recommended for the manufacturing of an HF pickup coil for installation on ITER. Further winding tests could be performed with other materials suitable for ITER, such as Glidcop, titanium, or molybdenum.

The first laser-cut sensor has been easily manufactured in SS-316 using industrial processes. This nonconventional prototype is considered to have yielded promising results, with the last remaining problem being that of adding a second winding layer and more turns in each layer (by reducing the wire thickness) so as to increase the effective area to values much closer to the ITER specifications. A possible solution exists, with two independent coaxial tubes, each one using a separate winding, which are finally soldered or brazed together. This design is now the subject of ongoing $R \& D$ work to verify its feasibility. Together with the laser-cut design, several other options are being explored, such as stacking of plane windings manufactured from a tungsten plate by electrical discharge machining and a coil made using the conventional spring manufacture. These further studies are reported separately in Ref. 20.

\section{MEASUREMENTS AND ANALYSIS OF THE ELECTRICAL CHARACTERISTICS OF THE PROTOTYPE PICKUP COILS}

The measurement requirements ${ }^{1,2}$ for HF magnetic instabilities in ITER indicate that this diagnostic system needs to be designed so as to have the capability of measuring MHD instabilities in the $10-$ to $500-\mathrm{kHz}$ frequency range and with amplitudes well below $\mid \delta B /$ $B_{\theta} \mid \sim 10^{-4}$ at the position of the sensors. When considering explicitly the estimated length $(>50 \mathrm{~m})$ and the specifications (including uncertainties on the manufacturing tolerances) currently available for the electrical characteristics of the signal cables up to the front-end electronics and the data acquisition modules in ITER, ${ }^{\mathrm{m}}$ it is not clear whether it is possible to use the simple approach where the system self-resonant frequency is mainly determined by the sensor inductance and the cable capacitance. Hence, the best design choices for the electrical parameters are given by increasing the sensor's self-resonance frequency, preferably above $\sim 5 \mathrm{MHz}$, reducing the sensor's self-inductance, preferably below $\sim 100 \mu \mathrm{H}$, and increasing as much as possible the effective area $(N A)_{E F F}>0.05 \mathrm{~m}^{2}$. Moreover, based on the operational experience acquired on the JET and TCV tokamaks, the coil's quality factor $Q$ needs to be $<30, Q$ being the ratio between the coil's selfresonance frequency and its bandwidth, so that the resonance band can be effectively resolved and its effect calibrated out from the measured raw data.

Here we consider the static, geometrical value as the direct-current $(\mathrm{dc})(\omega=0$ limit $)$ value of quantities that are intrinsically related to a frequency (time) behavior, such as the self-inductance $L_{S E L F}$ and selfcapacitance $C_{S E L F}$. The dc sensitivity of the sensor, i.e., its low-frequency-limit effective area $(N A)_{E F F}(\omega=0)$ is deduced separately either via measurements made at low frequencies $(<100 \mathrm{~Hz})$ with a Helmholtz coil or

\footnotetext{
${ }^{\mathrm{m}}$ Technical information provided courtesy of G. Vayakis, A. Encheva, and C. Walker (ITER Organization) and A. Perez (CRPP)
} 
calculated from the geometrical data giving the sensor's 3-D shape. The alternating-current (ac) characteristics of the HF coil, including the frequency-dependent effective area $(N A)_{E F F}(\omega)$, can be easily extracted from the measurement of the coil impedance ( $Z$-data) following the numerical technique described in the Appendix. These Z-data measurements were made with an impedance meter in the frequency range extending from $10 \mathrm{kHz}$ up to $13 \mathrm{MHz}$ (the minimum frequency and maximum frequency at which the internal calibration of the instrumentation used for the measurement is valid), so as to take into account the coil's self-resonance. Note that direct use (without analytic continuation through appropriate circuit modeling) of these $Z$-data measurements to frequencies well outside the $10-\mathrm{kHz}$-to-13$\mathrm{MHz}$ range may lead to wrong results because the measurements themselves are not valid and the numerical procedure used to interpret them is also not valid.

Six prototype coils were analyzed (as shown in Fig. 11), five of which with two variants:

1. CPla and CP1b: prototype version V0 (ITER design) with copper wire, and with (CP1a) and without (CP1b) the SS-316 bobbin

2. $C P 2 a$ and $C P 2 b$ : modified prototype version $\mathrm{V} 1$ with copper wire, and with (CP2a) and without (CP2b) the SS-316 bobbin

3. $C P 3 a$ and $C P 3 b$ : modified prototype version $\mathrm{V} 2$ with copper wire, and with (CP3a) and without (CP3b) the SS-316 bobbin

4. $C P 4 a$ and $C P 4 b$ : prototype version V0 (ITER design) with tungsten wire, and with (CP4a) and without (CP4b) the SS-316 bobbin

5. CP5a and CP5b: modified prototype version V1 with tungsten wire, and with (CP5a) and without (CP5b) the SS-316 bobbin

6. CP6a: laser-cut prototype, single coaxial tube in SS-316, without a SS-316 bobbin.

The SS-316 core used for these tests does not have the optional-as discussed in Sec. II-longitudinal cut: This choice was made so as to maximize the core's effect on the impedance measurements. The thermal shield was added for the electrical characterization of the conventional Mirnov-type prototypes (CP1, CP2, CP3, CP4, and CP5), but their electrical properties were not affected by it, as there is a deep longitudinal cut to allow for flux penetration; hence, these data will not be reported on here.

As taken from the ITER material handbook, ${ }^{18^{n}}$ the nominal electrical data for the conventional prototypes (CP1, CP2, CP3, CP4, and CP5) are: resistivity of the copper wire $\rho_{C U}=16.78 \times 10^{-9} \Omega \cdot \mathrm{m}$; resistivity of the tungsten wire $\rho_{W}=52.8 \times 10^{-9} \Omega \cdot \mathrm{m}$; resistivity of

\footnotetext{
${ }^{\mathrm{n}}$ See footnote k on p. 25.
}

the SS-316 core $\rho_{S S}=740 \times 10^{-9} \Omega \cdot \mathrm{m}$; dielectric constant $\varepsilon_{R}$ range for the RP PA12 polymer from $\varepsilon_{R}=3.0$ to $\varepsilon_{R}=4.2$ (as per the manufacturer's data sheet). The common geometrical data are: copper wire diameter and tungsten wire diameter are 0.6 and $0.5 \mathrm{~mm}$, respectively; 33 turns per layer with a separation between the two superposed layers of $0.6 \mathrm{~mm}$; nominal length for the inner side (first layer) and outer side (second layer) of the hexagonal (as built) coil are 18.4 and $22.6 \mathrm{~mm}$, respectively; the nominal length of the coil along its axis is $78 \mathrm{~mm}$. Hence, the nominal total length of the wire in this conventional design is $8.1 \mathrm{~m}$, and the dc nominal (geometrical) effective area $(N A)_{E F F}(\omega=0)$ is $0.0728 \mathrm{~m}^{2}$. The nominal electrical data for the laser-cut prototype in SS-316 are: The longitudinal thickness and gap between the equivalent wire is $1.6 \mathrm{~mm}$; the inner diameter and outer diameter of the circular cross section are 30 and $33 \mathrm{~mm}$, respectively; the number of turns is 16; the nominal coil length (i.e., without forcing the gap between the turns) is $49.6 \mathrm{~mm}$; the total wire length is $1.58 \mathrm{~m}$; the nominal $(\mathrm{dc})$ effective area $(N A)_{E F F}(\omega=0)$ is $0.0125 \mathrm{~m}^{2}$; the range of dielectric constant $\varepsilon_{\mathrm{R}}$ for Plexiglas is from 2.6 to 3.5. ${ }^{\circ}$

\section{V.A. Determination of DC Electrical Data}

Most of the dc electrical $(R, L$, and $C)$ characteristics of these prototypes can be evaluated analytically for the equivalent solenoid-like sensor of circular cross section with the same total geometrical area and wire length as the as-built hexagonal sensor. Neglecting skin-depth effects, this approach gives:

1. conventional design: 33 turns on two layers, nominal one-turn-area $A_{T U R N} \sim 11.03 \times 10^{-4} \mathrm{~m}^{2}$

2. conventional design, copper wire: self-inductance $L_{S E L F}=57.02 \mu \mathrm{H}, R=0.48 \Omega$

3. conventional design, tungsten wire: selfinductance $L_{S E L F}=60.66 \mu \mathrm{H}, R=2.18 \Omega$

4. conventional design, SS-316 core: self-inductance $L_{S E L F}=21.42 \mathrm{nH}, R=61.68 \mathrm{~m} \Omega$

5. conventional design, mutual inductance between SS-316 core and coil's winding: $M=279$ $\mathrm{nH}$

6. nominal coefficient of mutual coupling corewinding: $K=0.2441$

7. conventional design, capacitance core-coil in vacuum: $C=23.51 \mathrm{pF}$

8. conventional design, capacitance core-coil for PA12: $C=70.52 \mathrm{pF}$ to $C=98.72 \mathrm{pF}$

9. conventional design, model self-capacitance: $C_{\text {SELF }}=33.34 \mathrm{pF}$

${ }^{\circ}$ See http://www.csgnetwork.com/dieconstantstable.html (current as of Oct. 27, 2010). 
10. laser-cut design: 16 turns on one layer, nominal one-turn-area $A_{\text {TURN }} \sim 8.55 \times 10^{-4} \mathrm{~m}^{2}$

11. laser-cut design: self-inductance $L_{S E L F}=2.55$ $\mu \mathrm{H}, R=7.89 \mathrm{~m} \Omega$

12. laser-cut design, capacitance core-coil in vacuum: $C=28.02 \mathrm{pF}$

13. laser-cut design, capacitance core-coilfor Plexiglas: $C=72.84 \mathrm{pF}$ to $C=98.60 \mathrm{pF}$

14. laser-cut design, model self-capacitance: $C_{S E L F}=$ $15.20 \mathrm{pF}$.

The self-inductance of the SS-316 and its mutual inductance with the winding have been calculated using the approximations valid for two concentric, hollow (continuous for the core, multiturns for the winding), straight circular conductors, each one of finite dimensions, which gives the self-inductances of the SS-316 core:

$$
\begin{aligned}
L_{\text {SELF }}= & (\mu / 2 \pi) \times l_{\text {CORE }} \\
& \times\left[\log \left(2 l_{\text {CORE }} / R_{\text {OUT }}\right)-1\right. \\
& \left.\quad+g\left(R_{\text {INN }} / R_{\text {OUT }}, \mu_{1 r}\right)\right],
\end{aligned}
$$

and the mutual inductance of the SS-316 core with winding $\mathrm{M}_{\mathrm{CW}}$ :

$$
\begin{aligned}
M_{C W}= & \frac{\mu}{4 \pi} \sum_{j=T U R N} \frac{\sqrt{\left(R_{2, j}+R_{1}\right)^{2}+\left(R_{2, j}^{2}+l_{\text {CORE }}^{2}\right) / 2}}{l_{\text {CORE }} \times R_{2, j}} \\
& \times\left[l_{\text {CORE }}^{2}-R_{1}\left(2 R_{2, j}-R_{1}\right)\right] \\
& \times f\left(\frac{l_{\text {CORE }}}{R_{2, j}}, \frac{R_{1}}{R_{2, j}}, \frac{\Delta_{1}}{R_{1}}, \frac{\Delta_{2}}{R_{2, j}}, \frac{\mu_{1 r}}{\mu_{2 r}}\right),
\end{aligned}
$$

where $l_{C O R E}$ is the length and $R_{I N N}$ and $R_{O U T}$ are the inner radius and outer radius, respectively, of the hollow core (inside which there is a material with relative magnetic permeability $\mu_{1 r}$ with respect to that of the material surrounding the entire coil assembly, $\mu$, and that of the ceramic insulating, $\left.\mu_{2 r}\right) . R_{O U T}-R_{I N N}=\Delta_{1}$ is the thickness of the core; $R_{1}=\left(R_{I N N}+R_{\text {OUT }}\right) / 2, R_{2, j}$, and $\Delta_{2, j}$ are the mean radius and the equivalent thickness of each winding turn, respectively; $g(x)=O(1)$ and $f(x)=O(1)$ are two numerical fitting functions approximating the exact elliptic integrals and Heuman's Lambda functions entering the analytical formulation, and the sum is intended over all individual turns of the winding pack.

The self-capacitance $C_{S E L F}$ cannot be reliably calculated analytically due to the complexity of the actual coil's geometry. Hence we decided to try to calculate the self-capacitance of the prototype coil with FEMs, using the 2-D software packages COMSOL and FEMM ${ }^{\mathrm{p}}$ to

pFEMM software package: http://femm.foster-miller.net/wiki/ HomePage (current as of Oct. 27, 2010). compare the calculated values of $C_{S E L F}$. A complete 3-D calculation with COMSOL using the real, very thin and long wire exceeded our computational resources; hence, we considered a symmetrical 2-D case. As the differences between the 3-D geometry and the 2-D geometry are due to manufacturing and winding tolerances, we expect that the use of a 2-D simplified geometry will not change dramatically the value of $C_{S E L F}$. The 2-D COMSOL calculations were verified with more accurate 3-D results for the simpler case of a circular coil with diameter of $38 \mathrm{~mm}$, made from a long, thick wire of diameter of $2 \mathrm{~mm}$, with $N$ turns separated by $4 \mathrm{~mm}$, and changing the number of turns. This benchmark shows a good agreement between FEMM, COMSOL-2D, and COMSOL$3 \mathrm{D}$, typically within $5 \%$ for five turns and more, with the results from FEMM systematically larger than those given by COMSOL.

For the simplified 2-D geometry of the conventional prototypes, the dc value of the self-capacitance is $C_{S E L F}=$ $5.4 \mathrm{pF}$ and $C_{S E L F}=4.6 \mathrm{pF}$ using FEMM and COMSOL$2 \mathrm{D}$, respectively. Using the very simple model of a plane circular condenser to calculate the self-capacitance, we obtain the values of $C_{S E L F}=33.34 \mathrm{pF}$ and $C_{\text {SELF }}=59.22$ $\mathrm{pF}$ for the conventional prototype and the laser-cut prototype, respectively. When using the impedance and resonance frequency measurements (without the SS-316 core), we find that the self-capacitance for the conventional copper winding and tungsten winding are $C_{S E L F} \sim$ $28 \mathrm{pF}$ and $C_{S E L F} \sim 13 \mathrm{pF}$, respectively, with $C_{S E L F}<40$ $\mathrm{pF}$ for the laser-cut prototype. The analytical calculation of $C_{S E L F}$ (overestimating $C_{S E L F}$ by $\sim 20 \%$ ) actually gives a better estimate than the numerical results using FEMM and COMSOL (underestimating $C_{S E L F}$ by a factor of $\sim 6$ ).

\section{V.B. Determination of the AC Electrical Data}

The coil's self-resonance frequency $\omega_{0}=$ $1 /\left(L_{S E L F} C_{S E L F}\right)^{1 / 2}$ and bandwidth $\gamma=\left(\omega_{0}\right)^{2}\left(R C_{S E L F}+\right.$ $\left.P L_{S E L F}\right) / 2$ were determined from the measurement of the coil's impedance. Here $R$ and $P$ are the resistance and parallel admittance of each coil, respectively, evaluated at the resonant frequency. We have used a fixed $P=10^{-6}$ $\Omega^{-1}$ for all prototypes, taken as the inverse of the measured dc isolation resistance value, i.e., $P=1 / R_{I S O L-D C}$. Using the experimental values obtained with such a procedure for $L_{S E L F}$ and $C_{S E L F}$ and using such value for $P$, $R$ can then be determined from the measured bandwidth $\gamma$.

A best fit to the measured Z-data is also constructed from the numerical procedure giving the circuit parameters. As the length of the free wire in excess of the winding length is very small in our test equipment ( $\sim 10 \mathrm{~cm}$, compared to $\sim 8 \mathrm{~m}$ for the winding length), the best (and simplest) equivalent circuit that can be used to model the real coil has only 1 pole, i.e., the one shown in Fig. A.1a in the Appendix. This can be mathematically represented via rational functions of order $3 / 2$ (direct 
$Z$-data fit) or $5 / 2$ [when using the numerical approach leading to Eq. (A.8) in the Appendix]. Since the measured Z-data for all the CP2a, CP2b, CP3a, and CP3b prototypes are within the accuracy of the instrumentation used to obtain the $Z$-data, the electrical characteristics and the equivalent circuit model are taken to be the same for these designs; hence, the data for the $\mathrm{CP} 3 \mathrm{a}$ and $\mathrm{CP} 3 \mathrm{~b}$ prototypes are not shown.

Table I gives the values for the self-resonance frequency $\left(\omega_{0} / 2 \pi\right)$, the bandwidth $(\gamma / 2 \pi)$, and the quality factor $\left[Q=\omega_{0} /(2 \gamma)\right]$ for the conventional prototypes, with and without the SS-316 bobbin (without a longitudinal cut). The results using two rational function fits of different order $(3 / 2$ and $5 / 2)$ are essentially the same within the accuracy of the numerical procedure (estimated to be $<5 \%$ ). These data are not reported for the laser-cut coil (as mounted on a Plexiglas support) as its self-resonance frequency does not lie in the frequency range of the impedance measurements. Figure 14 shows the measured $Z$-data together with the fit values obtained using the two circuit models described above.

Figure 14a shows the measured and calculated impedance for the V0 prototypes with copper and tungsten wire, with and without the SS-316 bobbin, and Fig. 14b shows the same data for the laser-cut design. There is a very good agreement between the different $Z$-values, with only a minor discrepancy in the bandwidth, due to the choice of the same parallel admittance $P=10^{-6} \Omega^{-1}$ for all the different prototypes. This result shows that our model calculations can reproduce with a great accuracy the impedance measurements. The impedance of the lasercut prototype has a linear dependence on the frequency, indicating that this sensor is still an almost-pure inductor up to at least $13 \mathrm{MHz}$ (i.e., with $|Z| \sim \omega L$, with a small dc resistance $R=o(|\omega L|)$ in the frequency range of interest).

Figure $14 \mathrm{c}$ shows the measured $Z$-data for all the conventional prototypes up to $8 \mathrm{MHz}$. The self-resonance frequency ranges from $\omega_{0} / 2 \pi \sim 2.7 \mathrm{MHz}$ for the V1 prototype when using a copper wire without the SS-316 bobbin, to $\omega_{0} / 2 \pi \sim 6.4 \mathrm{MHz}$ for the V0 prototype when using a tungsten wire with the SS-316 bobbin. These data show that the differences in the grooving designs only very marginally affect the electrical characteristics of the various conventional prototypes, as expected. Conversely, the insertion of the SS-316 bobbin without a longitudinal cut increases the resonant frequency, moving it from values below $3 \mathrm{MHz}$ to values exceeding at least $4.5 \mathrm{MHz}$ for all the wire materials and grooving designs. The bandwidth also increases, and the quality factor reduces below the target value of $Q=30$.

The ac equivalent circuit model data have been evaluated in the frequency range between $10 \mathrm{kHz}$ and $13 \mathrm{MHz}$ for all the conventional prototypes, and the data are reported in Table II for the CP1, CP2, CP4, and CP5 cases, again showing the results for the two different rational function fits to the measured $Z$-data (as the results for the $\mathrm{CP} 3$ prototypes are very close to those for the $\mathrm{CP} 2$ ones, these are not tabulated for simplicity). For the case of the conventional Mirnov-type prototypes, the insertion of the SS-316 bobbin (without a longitudinal cut) essentially reduces the coil self-inductance. The SS316 bobbin acts as the one-turn capacitively loaded secondary of a transformer, the primary being the winding pack itself; this also contributes to increasing the effective ac coil resistance. Therefore, $L_{S E L F}$ reduces from $110<L_{S E L F}(\mu \mathrm{H})<150$ for the prototypes without the SS-316 bobbin to $35<L_{S E L F}(\mu \mathrm{H})<42$ for those with such metallic core. The coil's self-capacitance remains essentially the same with and without insertion of the SS-316 bobbin when using the same material for the winding pack. Conversely, there is a significant difference in $C_{S E L F}$ for the copper and tungsten prototypes: This can be associated to the much higher rigidity of tungsten, which makes it more difficult to reproduce

TABLE I

Frequency Characteristics of Each Individual Coil*

\begin{tabular}{|c|l|l|c|c|c|}
\hline Coil & $\begin{array}{c}\text { Wire } \\
\text { Material }\end{array}$ & \multicolumn{1}{|c|}{ Comment } & $\begin{array}{c}\omega_{0} / 2 \pi \\
(\mathrm{MHz})\end{array}$ & $\begin{array}{c}\gamma / 2 \pi \\
(\mathrm{kHz})\end{array}$ & $\begin{array}{c}\omega_{0} /(2 \gamma) \\
\text { Quality Factor }\end{array}$ \\
\hline CP1a & Copper & Prototype version V0, no SS-316 core & 2.83 & $22.01 / 24.27$ & $64.19 / 58.27$ \\
CP1b & Copper & Prototype version V0, SS-316 core & 4.75 & $134.75 / 134.35$ & $17.63 / 17.67$ \\
CP2a & Copper & Prototype version V1, no SS-316 core & 2.68 & $22.84 / 26.07$ & $58.73 / 51.54$ \\
CP2b & Copper & Prototype version V1, SS-316 core & 4.49 & $150.35 / 149.80$ & $14.94 / 14.94$ \\
CP4a & Tungsten & Prototype version V0, no SS-316 core & 4.00 & $33.16 / 35.40$ & $60.26 / 56.41$ \\
CP4b & Tungsten & Prototype version V0, SS-316 core & 6.41 & $169.43 / 150.45$ & $14.94 / 14.94$ \\
CP5a & Tungsten & Prototype version V1, no SS-316 core & 3.67 & $33.00 / 33.39$ & $55.61 / 54.94$ \\
CP5b & Tungsten & Prototype version V1, SS-316 core & 5.93 & $196.79 / 201.95$ & $15.07 / 14.54$ \\
\hline
\end{tabular}

*Resonance $\left(\omega_{0} / 2 \pi\right)$, bandwidth $(\gamma / 2 \pi)$, and quality factor $[Q=\omega /(2 \gamma)]$, evaluated from the best numerical fit to the measured Z-data; a SS-316 core without a longitudinal cut was used to maximize its effect on the sensor's impedance.

"The "/" separate the results obtained using two different rational function fits (model1 with a 3/2 polynomial order and model 2 with a $5 / 2$ polynomial order, respectively, as shown in Fig. 14a). 
Zdata vs. Zmodel for ITER prototype HF coils

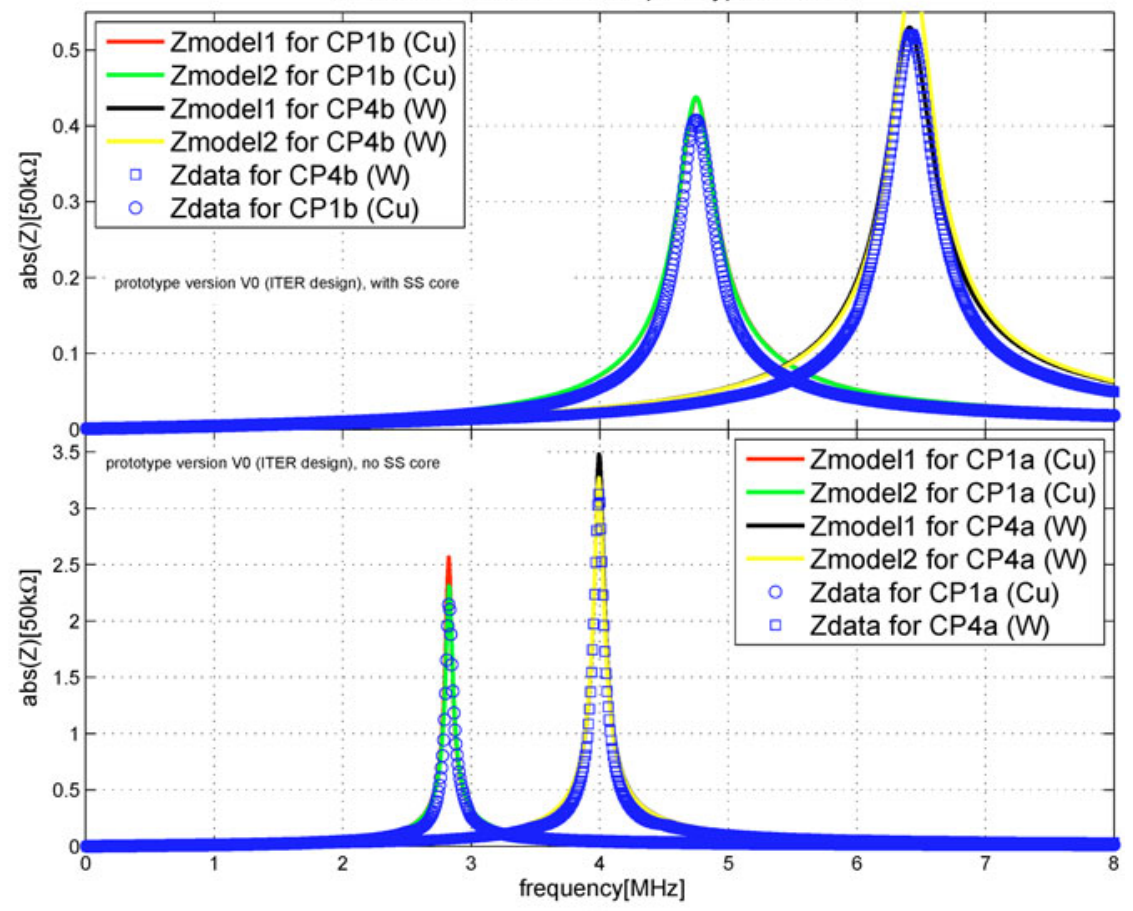

(a)



(b)

Figs. 14a and 14b. (a) Measured and equivalent circuit model Z-data as evaluated using two different rational function fits for some of the ITER HF coil prototypes of conventional design (model1 with a $3 / 2$ polynomial order and model 2 with a 5/2 polynomial order, respectively); the results obtained using these two different rational function fits are essentially the same. The codenames $\operatorname{CPx}(\mathrm{a}, \mathrm{b})$ for the various prototypes are explained in the main body of the text. (b) Measured and calculated $Z$-data for the laser-cut, nonconventional prototype: Here a simple $R$, $L$ circuit was used as the impedance increases linearly as function of the frequency. 


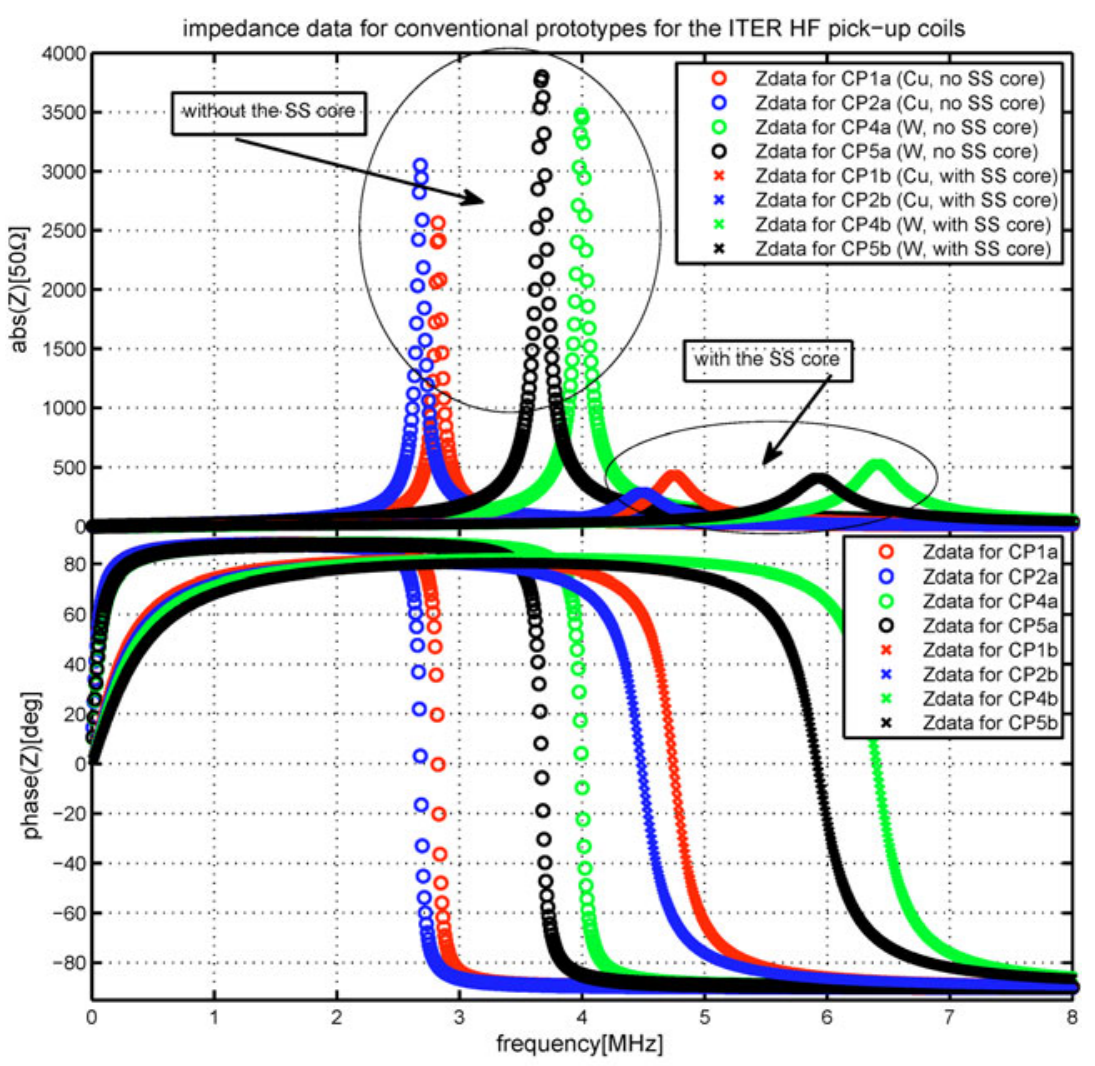

(c)

Fig. 14c. Measured impedance data for the ITER HF coil prototypes of conventional design: Note that the self-resonance frequency (and the bandwidth) increases when inserting the SS-316 core without the longitudinal cut, and it is higher when using tungsten because of the lower self-capacitance of the winding pack; on the other hand, the different designs for the grooving have very little impact on the self-resonance frequency and bandwidth.

"exactly" the same hexagonal shape on the $2 \times 33$ individual layers (see Sec. IV). The calculated quality factor is in the range $50<Q<65$ for the prototypes without the SS-316 bobbin and reduces to values in the range $15<Q<20$ for those with the SS-316 bobbin, because the coil bandwidth increases relatively more than the self-resonance frequency.

For the laser-cut prototype the ac values of the resistance and inductance have been calculated for frequencies $<10 \mathrm{kHz}$, where $|Z|$ is almost constant, and frequencies $>1 \mathrm{MHz}$, where phase $(Z) \sim 90 \mathrm{deg}$, respectively, giving $R \sim 595 \mathrm{~m} \Omega$ and $L_{S E L F}=3.90 \mu \mathrm{H}$. This value of $L_{S E L F}$ then gives an upper limit for $C_{S E L F}$, as the self-resonance frequency is not found up to $13 \mathrm{MHz}$, hence, $C_{S E L F}<39 \mathrm{pF}$.

\section{V.C. Measurement of the Effective Area}

Producing a sensor with a sufficiently large value for $(N A)_{E F F}$ is essential to achieve a good signal-tonoise ratio for the measurement of the MHD instabilities as $(N A)_{E F F}$ acts as a "gain" for the voltage measured at the HF pickup coil: $V_{M E A S}(\omega)=(N A)_{E F F}(\omega) \times i \omega \times$ $B_{M E A S}(\omega)$. It is clear that using the frequency-dependent value for $(N A)_{E F F}(\omega)$ is essential to obtain a correct measurement for the amplitude of the instabilities detected with any array of HF magnetic sensors. Based on our experience on the JET tokamak, and following the derivation presented in the Appendix, to evaluate the frequency-dependent $(N A)_{E F F}(\omega)$ we have taken the ansatz that this can be separated in a dc-only sensitivity $(N A)_{E F F}(\omega=0)$, corresponding to the geometrical value of the effective area, and a frequency-dependent transfer function $H_{A C}(\omega)$ deduced from the ac impedance measurements.

The sensor's effective area $(N A)_{E F F}$ has been directly measured from the dc range (as low as $20 \mathrm{~Hz}$ ) up to $3 \mathrm{MHz}$ with a Helmholtz coil. The frequency response of this equipment (current and voltage measurement, impedance) is fully calibrated as we measure directly the driven current via a shunt and the driven voltage via a current transformer, hence automatically removing the effect of any possible self-capacitance of the Helmholtz coils themselves. Note that the measurement of 
TABLE II

Electrical Circuit Data for the Various Conventional Prototypes*

\begin{tabular}{|c|c|c|c|c|c|c|c|c|}
\hline & \multicolumn{4}{|c|}{ Without SS-316 Core } & \multicolumn{4}{|c|}{ With SS-316 Core } \\
\hline & \multicolumn{2}{|c|}{ Copper Wire } & \multicolumn{2}{|c|}{ Tungsten Wire } & \multicolumn{2}{|c|}{ Copper Wire } & \multicolumn{2}{|c|}{ Tungsten Wire } \\
\hline & $\mathrm{CP} 1 \mathrm{a}$ & $\mathrm{CP} 2 \mathrm{a}$ & $\mathrm{CP} 4 \mathrm{a}$ & $\mathrm{CP} 5 \mathrm{a}$ & $\mathrm{CP} 1 \mathrm{~b}$ & $\mathrm{CP} 2 \mathrm{~b}$ & $\mathrm{CP} 4 \mathrm{~b}$ & $\mathrm{CP} 5 \mathrm{~b}$ \\
\hline$L(\mu \mathrm{H})$ & 112.49 & 154.44 & 115.40 & 148.64 & 41.44 & 34.28 & 34.72 & 36.44 \\
\hline$P(1 / \mathrm{M} \Omega)$ & 1.00 & 1.00 & 1.00 & 1.00 & 1.00 & 1.00 & 1.00 & 1.00 \\
\hline Model1: $R(\Omega)$ & 27.12 & 37.55 & 39.69 & 49.89 & 68.63 & 63.81 & 71.97 & 88.28 \\
\hline Model2: $R(\Omega)$ & 30.32 & 43.79 & 42.94 & 50.63 & 68.43 & 63.60 & 63.67 & 91.55 \\
\hline Model1: $C(\mathrm{pF})$ & 28.21 & 22.79 & 13.75 & 12.65 & 27.08 & 36.61 & 17.76 & 19.77 \\
\hline Model2: $C(\mathrm{pF})$ & 28.14 & 22.72 & 13.77 & 12.66 & 27.11 & 36.87 & 17.62 & 19.76 \\
\hline fres1 $(\mathrm{MHz})$ & 2.83 & 2.68 & 4.00 & 3.67 & 4.75 & 4.49 & 6.41 & 5.93 \\
\hline $\mathrm{BW} 1(\mathrm{kHz})$ & 22.01 & 22.84 & 33.16 & 33.00 & 134.75 & 150.30 & 169.43 & 196.79 \\
\hline Qfact1 & 64.19 & 58.73 & 60.26 & 55.61 & 17.63 & 14.94 & 18.91 & 15.07 \\
\hline fres $2(\mathrm{MHz})$ & 2.83 & 2.69 & 3.99 & 3.67 & 4.75 & 4.48 & 6.43 & 5.93 \\
\hline $\mathrm{BW} 2(\mathrm{kHz})$ & 24.27 & 26.07 & 35.40 & 33.39 & 134.35 & 149.80 & 150.45 & 201.95 \\
\hline Qfact2 & 58.27 & 51.54 & 56.41 & 54.94 & 17.67 & 14.94 & 21.39 & 14.54 \\
\hline
\end{tabular}

*Evaluated using the same rational function fits to the measured Z-data (model1 with a 3/2 polynomial order and model2 with a $5 / 2$ polynomial order, respectively, as shown in Fig. 14a) used to obtain the results shown in Table I: The main circuit elements are essentially the same with these two calculations.

$(N A)_{E F F}(\omega)$ above a cutoff frequency $\omega_{H} \sim 10 \mathrm{kHz}$ is difficult to interpret directly without prior knowledge of the sensor impedance $Z(\omega)$, as one is in fact measuring the combined effect of $(N A)_{E F F}(\omega)$ and $Z(\omega)$. A practical value for $\omega_{H}$ is the frequency point where the phase of $Z(\omega)$ is no longer constant (i.e., the sensor is not anymore a "pure dc resistor," so that $R \gg L \omega_{H}$ ) but starts increasing as a linear function of $\omega$ (i.e., the sensor behaves as an inductor in series with a smaller dc resistance), hence $\omega_{H} \sim \alpha R / L$, with $\alpha=1 \rightarrow 5$ depending on the actual sensor's design.

For the standard Helmholtz coil layout, the magnetic field $B_{H E L M}$ was described in Sec. III. This nominal value of $B_{H E L M}(\omega=0)$ on the horizontal axis of the Helmholtz system agrees within a $5 \%$ difference with the 2-D numerical result obtained with COMSOL. The voltage measured across the prototype under test is then given by:

$$
V_{M E A S}(\omega)=(N A)_{E F F}(\omega) \times i \omega \times B_{H E L M}(\omega)
$$

and

$$
V_{\text {STRAY }}(\omega)=(N A)_{\text {STRAY }}(\omega) \times i \omega \times B_{H E L M}^{(S T R A Y)}(\omega) .
$$

In Eq. (1), $V_{\text {MEAS }}$ and $V_{\text {STRAY }}$ give the measurements of the effective $(N A)_{E F F}$ and of the stray area $(N A)_{S T R A Y}$ for the test prototype, the stray area being defined as the area perpendicular to the longitudinal axis of the Mirnov coil. For the measurement of the stray area, two different setups were used: the standard one with the axis of Helm- holtz system perpendicular to the axis of the prototype Mirnov coil, and a second one using a four-turn winding across the longitudinal axis of the test prototype, which was in this case aligned with the axis of the Helmholtz system. This additional winding for the measurement of the stray area was insulated from the prototype's main ceramic body and winding package using cellotape, and the stray voltage $V_{\text {STRAY }}$ was measured across this additional winding. Using both measurement setups, the measured stray area is much lower than the effective area at all frequencies up to $3 \mathrm{MHz}$, not exceeding $(N A)_{S T R A Y} /$ $(N A)_{E F F} \leq 0.005$ for the prototypes made with a copper wire (irrespective of the grooving design; i.e., all data are within a $1 \%$ scatter from the mean value), whereas $(N A)_{S T R A Y} /(N A)_{E F F} \sim 0.01$ for those made with a tungsten wire, where the scatter between the different prototypes is also larger, $\sim 5 \%$.

Using the geometrical data for the prototypes given in Sec. V.A, the nominal dc effective area is $(N A)_{E F F-D C}=0.0728 \mathrm{~m}^{2}$ and $(N A)_{E F F-D C}=0.0125 \mathrm{~m}^{2}$ for the Mirnov-type prototype and the laser-cut prototype, respectively. The presence of the SS-316 bobbin (without a longitudinal cut) affects the HF value of $(N A)_{E F F}(\omega)$, since this continuous metallic element acts as the secondary of a transformer, the primary being the winding pack itself. Table III and Fig. 15 show the values of $(N A)_{E F F}(\omega)$ in the frequency range from $20 \mathrm{~Hz}$ to $3 \mathrm{MHz}$ for the ITER original design, with copper and tungsten wire, with or without the SS-316 bobbin, and for the laser-cut sensor (these data are not shown for the other grooving design as they are all very similar, within 
TABLE III

Effective Area for All the HF Sensor Prototypes Considered*

\begin{tabular}{|r|c|c|c|c|c|}
\hline $\begin{array}{c}\text { Frequency } \\
(\mathrm{kHz})\end{array}$ & $\begin{array}{c}(N A)_{E F F}\left(\mathrm{~m}^{2}\right) \\
\mathrm{Cu}, \text { no SS-316 }\end{array}$ & $\begin{array}{c}(N A)_{E F F}\left(\mathrm{~m}^{2}\right) \\
\mathrm{Cu}, \text { with SS-316 }\end{array}$ & $\begin{array}{c}(N A)_{E F F}\left(\mathrm{~m}^{2}\right) \\
\mathrm{W}, \text { no SS-316 }\end{array}$ & $\begin{array}{c}(N A)_{E F F}\left(\mathrm{~m}^{2}\right) \\
\text { W, with SS-316 }\end{array}$ & $\begin{array}{c}(N A)_{E F F}\left(\mathrm{~m}^{2}\right) \\
\text { Laser-Cut }\end{array}$ \\
\hline 0.02 & $6.0086 \mathrm{E}-02^{\mathrm{a}}$ & $6.0086 \mathrm{E}-02$ & $6.0057 \mathrm{E}-02$ & $6.0057 \mathrm{E}-02$ & $1.0809 \mathrm{E}-02$ \\
0.05 & $6.0454 \mathrm{E}-02$ & $6.0237 \mathrm{E}-02$ & $6.0484 \mathrm{E}-02$ & $6.0842 \mathrm{E}-02$ & $1.1764 \mathrm{E}-02$ \\
0.10 & $6.0555 \mathrm{E}-02$ & $6.0555 \mathrm{E}-02$ & $6.0555 \mathrm{E}-02$ & $5.9518 \mathrm{E}-02$ & $1.0733 \mathrm{E}-02$ \\
0.20 & $6.0783 \mathrm{E}-02$ & $6.0783 \mathrm{E}-02$ & $6.0783 \mathrm{E}-02$ & $6.0783 \mathrm{E}-02$ & $1.1337 \mathrm{E}-02$ \\
0.50 & $6.0643 \mathrm{E}-02$ & $6.0821 \mathrm{E}-02$ & $6.0643 \mathrm{E}-02$ & $6.1643 \mathrm{E}-02$ & $1.1596 \mathrm{E}-02$ \\
1.00 & $6.0926 \mathrm{E}-02$ & $6.0826 \mathrm{E}-02$ & $6.0926 \mathrm{E}-02$ & $6.2998 \mathrm{E}-02$ & $1.2078 \mathrm{E}-02$ \\
2.00 & $6.1046 \mathrm{E}-02$ & $6.0975 \mathrm{E}-02$ & $5.9396 \mathrm{E}-02$ & $5.9327 \mathrm{E}-02$ & $1.1427 \mathrm{E}-02$ \\
5.00 & $5.9625 \mathrm{E}-02$ & $6.0623 \mathrm{E}-02$ & $5.9625 \mathrm{E}-02$ & $6.0623 \mathrm{E}-02$ & $1.1163 \mathrm{E}-02$ \\
10.00 & $5.6897 \mathrm{E}-02$ & $5.9896 \mathrm{E}-02$ & $5.6897 \mathrm{E}-02$ & $5.6901 \mathrm{E}-02$ & $1.1429 \mathrm{E}-02$ \\
20.00 & $5.2963 \mathrm{E}-02$ & $5.6963 \mathrm{E}-02$ & $4.5117 \mathrm{E}-02$ & $4.8524 \mathrm{E}-02$ & $1.1617 \mathrm{E}-02$ \\
50.00 & $4.6438 \mathrm{E}-02$ & $4.8164 \mathrm{E}-02$ & $2.7863 \mathrm{E}-02$ & $2.8899 \mathrm{E}-02$ & $1.1424 \mathrm{E}-02$ \\
100.00 & $3.2500 \mathrm{E}-02$ & $3.3500 \mathrm{E}-02$ & $1.6250 \mathrm{E}-02$ & $1.6750 \mathrm{E}-02$ & $1.1474 \mathrm{E}-02$ \\
150.00 & $1.9760 \mathrm{E}-02$ & $1.8836 \mathrm{E}-02$ & $8.8921 \mathrm{E}-03$ & $9.4179 \mathrm{E}-03$ & $1.1508 \mathrm{E}-02$ \\
200.00 & $7.1715 \mathrm{E}-03$ & $4.1715 \mathrm{E}-03$ & $2.8686 \mathrm{E}-03$ & $1.6686 \mathrm{E}-03$ & $1.1566 \mathrm{E}-02$ \\
500.00 & $5.0884 \mathrm{E}-03$ & $3.7060 \mathrm{E}-03$ & $2.5442 \mathrm{E}-03$ & $1.8530 \mathrm{E}-03$ & $6.9515 \mathrm{E}-03$ \\
1000.00 & $3.5649 \mathrm{E}-03$ & $3.4199 \mathrm{E}-03$ & $1.1883 \mathrm{E}-03$ & $8.5497 \mathrm{E}-04$ & $1.5927 \mathrm{E}-03$ \\
1500.00 & $3.2050 \mathrm{E}-03$ & $3.2050 \mathrm{E}-03$ & $1.6025 \mathrm{E}-03$ & $1.6025 \mathrm{E}-03$ & $1.0586 \mathrm{E}-03$ \\
2000.00 & $3.0020 \mathrm{E}-03$ & $3.0020 \mathrm{E}-03$ & $1.5010 \mathrm{E}-03$ & $1.5010 \mathrm{E}-03$ & $8.0229 \mathrm{E}-04$ \\
2500.00 & $2.6835 \mathrm{E}-03$ & $2.7302 \mathrm{E}-03$ & $1.0063 \mathrm{E}-03$ & $1.0921 \mathrm{E}-03$ & $6.0667 \mathrm{E}-04$ \\
3000.00 & $2.4772 \mathrm{E}-03$ & $2.4349 \mathrm{E}-03$ & $4.9544 \mathrm{E}-04$ & $6.9569 \mathrm{E}-04$ & $6.1329 \mathrm{E}-04$ \\
\hline
\end{tabular}

*For prototypes made using the ITER design (prototype version V0) with copper $(\mathrm{Cu})$ and tungsten (W) wire, with and without inserting the SS-316 core, and the laser-cut nonconventional design; these measurements were made with the Helmholtz coil assembly and combined with the sensor impedance data, as described in the Appendix.

${ }^{a}$ Read as $6.0086 \times 10^{-2}$.

an $\sim 10 \%$ difference). These data are obtained by combining the Helmholtz system with the sensor's impedance measurements (the latter being extrapolated to frequencies $\omega<2 \pi \times 20 \mathrm{kHz}$ by analytic continuation through a circuit modeling based on an almost purely resistive sensor: $R \gg \omega L_{S E L F}$ ).

In general, there is a rather good agreement (within $10 \%)$ between the measurement of $(N A)_{E F F}(\omega)$ for frequencies $<10 \mathrm{kHz}$ and the geometrical data. We suggest that only the imperfections in the mechanical assembly of the sensors are the cause for such differences. For the Mirnov-type sensors, we note that changing the wire material has essentially no effect on $(N A)_{E F F}(\omega)$ but for frequencies in the range from $100 \mathrm{kHz}$ to $1 \mathrm{MHz}$ : This is clearly seen in Fig. 15a, and we suggest that this is due to the difficulties we encountered in winding the tungsten wire around the nominal hexagonal cross section of the prototype Mirnov coil, which resulted in a skewed quasicircular shape and a nonperfect alignment of the different turns for the winding pack made with tungsten. We note the absolute reduction in $(N A)_{E F F}(\omega)$ above $\sim 20$ $\mathrm{kHz}$ when using the SS-316 bobbin without the longitudinal cut. This reduction is of the order of a factor $\sim 5$, and it is due to a screening of the magnetic flux by the metallic core.

\section{V.D. Conclusions on the Measurement and Analysis of the Electrical Characteristics}

The electrical characteristics of all test prototypes have been evaluated in the dc and ac frequency ranges, with the data obtained in these two regimes in sufficiently good agreement, with the notable exception of $C_{S E L F}$. These sensors behave as expected in terms of their main electrical properties (frequency, bandwidth, self-inductance, effective area), and in general our model calculations agree very well with the measurements, which will allow us to predict with confidence the electrical properties of future prototypes without the need of actually building and testing them individually, hence clearly facilitating any R\&D work. The measurements of $(N A)_{E F F}(\omega)$ have shown that the geometrical dc value is a sufficiently correct estimate of the actual value of the effective area only when the winding pack follows closely the nominal shape of the coil itself. The higher self-resonant frequency for the prototypes made with tungsten wire is due to their lower self-capacitance. The tungsten wire is also more resistive but slightly less inductive than the copper wire, which further contributes to giving a higher self-resonance frequency. The often slightly lower self-inductance and larger stray area 
(a)

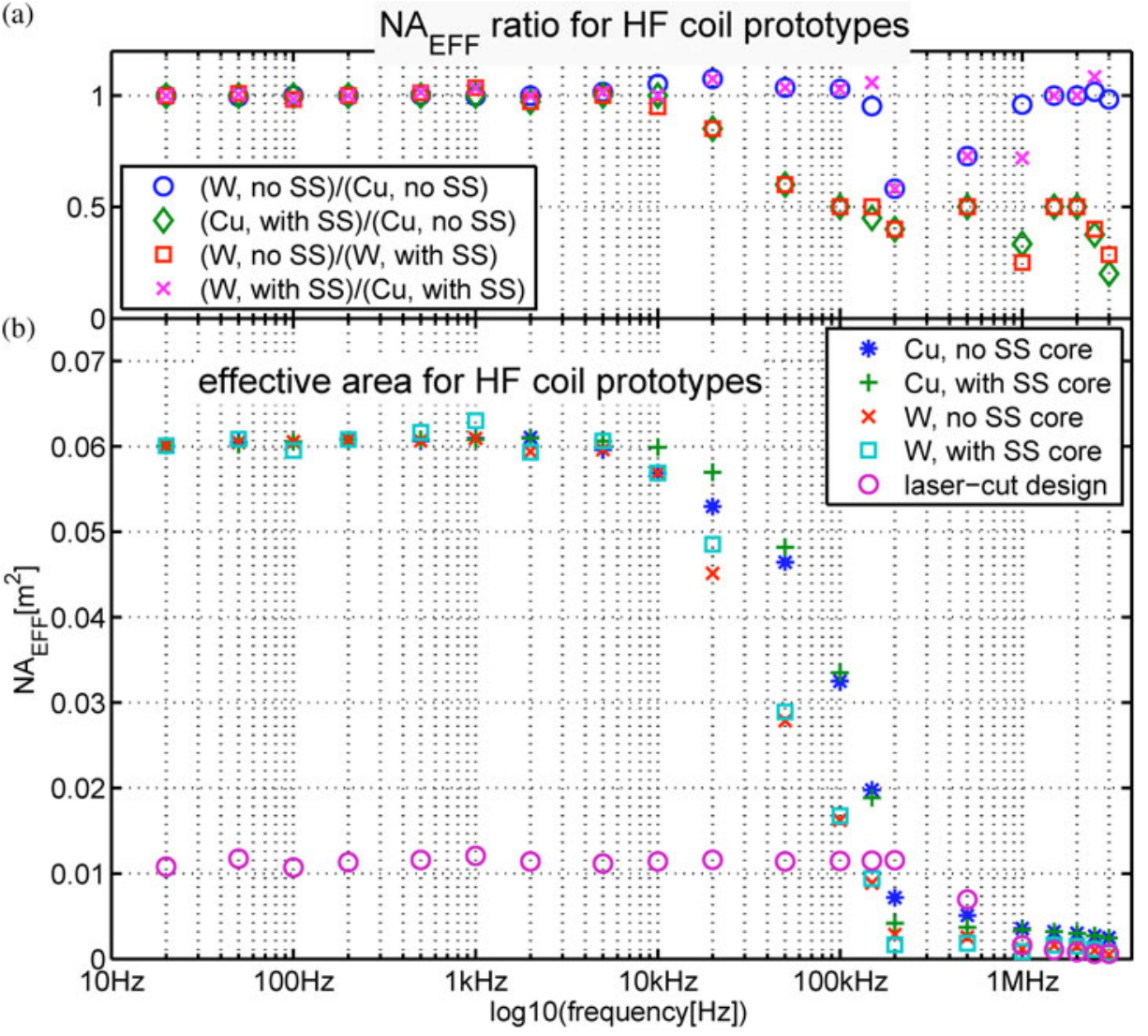

Fig. 15. In the bottom frame (b), the values of $(N A)_{E F F}(\omega)$ for the HF pickup coil prototypes obtained combining the Helmholtz and impedance measurements. In the top frame (a), the ratios between some of the $(N A)_{E F F}$ measurements for the conventional Mirnov-type sensors.

for the tungsten winding pack can be attributed to a very loose fit of the intended hexagonal shape of the polyamide body, due to the lower ductility of tungsten. Hence, when suitable and entirely practical design choices are made, the electrical properties of the sensors can be easily made to satisfy the required measurement performance.

The data for all the conventional Mirnov-type coils wound with copper wire have been found to be very similar irrespective of the grooving design, as they are all within an $\sim 10 \%$ scatter from the mean value. This can easily be understood as the differences in the winding alignment due to the differences in the grooving are too small to affect the electrical properties. Similarly, the different materials used for the winding package (copper versus tungsten) do not affect these properties only if the intended hexagonal shape of the test prototype is sufficiently adhered to, which also explains the larger scatter $(\sim 20 \%)$ observed in the measurements for the various prototypes made with tungsten.

For the conventional Mirnov-type prototypes, insertion of the SS-316 bobbin (without a longitudinal cut) reduces the coil self-inductance and increases the selfresonance frequency, with a clear beneficial effect on the sensor's electrical performance. However, this must be traded off against the reduction in $(N A)_{E F F}(\omega)$ above $\sim 20 \mathrm{kHz}$ and an increased mechanical risk to the assembly due to the possible onset of eddy currents on such a continuous metallic sheet. This effect is unavoidable for a Mirnov-type coil that is designed as the ITER prototype (individual ceramic "combs" or spacers carved out from a single ceramic body, both options being mounted on a metallic core) and can be associated to the shielding of magnetic flux by the SS-316 bobbin.

Finally, whereas the self-resonance frequency of the laser-cut prototype is found to be very high $(>13 \mathrm{MHz})$, which is very interesting for our applications, its effective area is clearly not sufficient (only $\sim 0.01 \mathrm{~m}^{2}$ ). We have also found a slightly bigger level of electrical pickup noise during the effective area measurement, and a slight increase in the stray area with respect to the other Mirnovtype sensors, mostly likely due to some manufacturing imperfections. Further optimization of the design for the laser-cut sensor is needed in order to manufacture a prototype with $(N A)_{E F F}$ comparable to that of the conventional sensor. By increasing the effective area, one will need to make sure that the stray area and the noise pickup 
will remain weak enough, so as to not decrease the quality and the overall sensitivity of the signal.

\section{CONCLUSIONS AND AN OUTLOOK TOWARD FUTURE WORK}

This work presents the results of the analysis of the assembly of the winding pack and of the electrical characteristics of various designs for the prototype for the HF magnetic pickup coils of Mirnov-type design appropriate for ITER. This work started with a FEM analysis of the ITER design for this sensor, which indicated that the differential thermal heating in the assembly could be the cause of excessive tensional strain between the ceramic body of the sensor and the winding pack itself, which may lead to breakages of the thin edges in the ceramic grooving.

Hence, the physical and mechanical characteristics of the prototype ITER HF pickup coils were analyzed with particular attention to the assembly process for the winding pack, by using different guiding grooves on the mechanical support and materials for the wire itself. The conventional prototypes were realized using the socalled RP technology to manufacture the support for the winding pack instead of the ceramic material envisaged in the ITER design of the HF pickup coil, which considerably reduced the time and costs of this test program.

Three different conventional designs for the guiding grooves were tested to assess the strength and manufacturability of the winding pack assembly and were wound with a copper wire and a tungsten wire: (a) the ITER design, i.e., a body with straight guiding grooves; (b) a body with modified slanted, but still straight, grooving; and (c) a body with modified grooving using passing holes. Our test program has demonstrated that these winding processes are not adequate, particularly because the tungsten wire currently foreseen to be used in ITER can easily be broken during the many operations necessary to assemble the winding pack. On the other hand, the electrical properties of the sensors can be relatively easily made to satisfy the intended measurement performance, with the only remaining concern that of using a SS-316 core with or without a longitudinal cut, where a larger self-resonance frequency has to be traded off with reduced strength and a slightly lower effective area. Hence, if the well-proven Mirnov-type technology is to be used for the ITER HF magnetic sensors, it is suggested that a revision of the current design should occur and that a tradeoff should be accepted on the sensor effective area, whereby by using a more standard Mirnov-type coil with much thicker ceramic grooves - and/or one single winding layer-wound with a wire other than tungsten (such as Glidcop), one would effectively reduce (even possibly halve) the effective area $(N A)_{E F F}$ in exchange for a much more mechanically robust sensor.

Following these initial tests, a new conceptual design for a nonconventional Mirnov-type pickup coil was developed, using laser cutting of a SS-316 hollow tube to produce one single helicoidal layer. This design completely avoids the need for a ceramic body with a complex network of thin guiding grooves, hence simplifying the manufacturing and assembly process. This prototype is promising but clearly needs further work to add a second coaxial layer and/or more turns on each layer so as to increase its effective area to values closer to the ITER specifications.

A further approach to reducing the risks of developing the HF magnetic diagnostic system for ITER is to develop a prototyping program for alternative design concepts, using completely different technologies. Using different technologies is also in line with risk-mitigation strategies that aim to reduce the "common mode" failure of sensors due to environmental constraints, such as (possibly highly localized) neutron and radiation fluxes and thermal gradients. Finally, there is a need to integrate the results from this prototyping work with the continuously evolving engineering and installation constraints of the ITER vacuum vessel. One major constraint is in fact the availability of space, as it is currently intended to position the individual HF magnetic sensors either in a very limited number of diagnostic cutouts in the blanket modules and around the port structures, where one can accommodate a sensor occupying a volume of the order of $\sim 50 \times 50 \times 50 \mathrm{~mm}$, or between the inner vessel wall and the blanket modules, where more positions are available but the thickness of the sensor becomes the most limiting factor, as it has to be $<7 \mathrm{~mm}$. $^{\mathrm{q}}$ Therefore, it is also clear that different designs for these sensors will likely have to be used. In this respect, the low-temperature co-fired ceramic technology ${ }^{21}$ is a very good starting point, as it is already an industry standard widely used for high-temperature and/or high-vacuum applications and also seems to be a particularly suitable candidate as far as volume occupation is concerned. The similar high-temperature co-fired ceramic technology has indeed been previously used for the manufacturing of low-frequency pickup coils on LHD (Ref. 22). Some initial prototyping tests have also been performed for the low-frequency magnetic sensors to be used for equilibrium reconstruction in ITER (Ref. 23). All these prototyping activities are the subject of ongoing $R \& D$ work at CRPP-EPFL, and the initial results are reported in Refs. 20, 21, and 24.

\section{APPENDIX}

\section{NUMERICAL DETERMINATION OF THE ELECTRICAL CHARACTERISTICS OF A HIGH-FREQUENCY MAGNETIC PICKUP COIL}

The transfer function for a system constituted of an in-vessel magnetic sensor, a set of cables, and the data

\footnotetext{
${ }^{\mathrm{q}}$ See footnote $\mathrm{m}$ on p. 35 .
} 
acquisition electronics cannot in general be measured directly because of access restriction to the inside of the machine. Therefore, a remote technique has been developed that relies upon our ability to measure the complex impedance of the probe-cable circuit as a function of frequency. ${ }^{4,25} \mathrm{~A}$ model of the equivalent probe-cable circuit incorporating the known circuit elements then allows the transfer function to be determined from a parameterized fit to the measured impedance. This technique, originally presented in Ref. 4, is based on the work of J-M. Moret on the TCV tokamak at CRPP (Ref. 25) and makes use of our knowledge of the electrical structure of a circuit with an HF magnetic sensor. Basically, we measure the circuit impedance versus frequency and then use the circuit structure to determine the ac component of the overall transfer function, using then either geometrical data or measurements made with a Helmholtz coil to deduce the dc "gain" of the sensor, i.e., its effective area. The ac response curves are in general nontrivial because the probe circuits are nonideal; the simple response function of an HF magnetic sensor,

$$
V_{M E A S}(\omega)=(N A)_{E F F}(\omega) \times i \omega B_{M E A S}(\omega),
$$

describes the voltage induced across the probe by a timevarying magnetic field, but the probe has a finite selfinductance and resistance, and it is connected (in principle) to a long set of cables, each with its own inductance and capacitance. Circuit resonances therefore alter the voltage that reaches the front-end electronics compared to that measured at the probe itself.

For ac circuits it is convenient to work in the Laplace $(s=i \omega)$ frequency-domain space. Here the output signal $S$ (in digitizer counts) of the front-end electronics is related to the rate of change of the magnetic field $\partial B_{M E A S} / \partial t$ along the probe axis via:

$$
\begin{aligned}
S(\text { counts }) & =H_{D}(s)(\text { count } / \mathrm{V}) \times V_{M E A S}(s)(\mathrm{V}) \\
& =H_{D}(s) \times H_{P}(s) \times B_{M E A S}(s), \quad(A
\end{aligned}
$$

where

$$
\begin{aligned}
H_{D}(s)= & \text { ac transfer function of the data acquisition } \\
& \text { electronics } \\
H_{P}(s)= & \text { complete ac transfer function of the probe- } \\
& \text { cable section, including (implicitly in the } \\
& \text { numerical procedure, but not explicitly } \\
& \text { in the mathematical formulation presented } \\
& \text { in Ref. } 4) \text { the term } s \times(N A)_{E F F}(s) \text { evalu- } \\
& \text { ated using the geometrical value for } \\
& (N A)_{E F F}, \\
B_{M E A S}= & \text { magnetic fluctuation being measured at the } \\
& \text { probe. }
\end{aligned}
$$

Note that the "geometrical" term $s \times(N A)_{E F F}(s)$ can be, and often is, included numerically in $H_{p}(s)$ for math- ematical simplicity, but this in fact is usually deduced separately (and then verified directly against the fit results) from Helmholtz coil measurements or geometrical data. The two transfer functions can individually be represented in the frequency domain as $H(s)=B(s) / A(s)$, where $B(s)$ is the output and $A(s)$ is the input. The calibration problem then reduces to the problem of determining $H_{D}(s)$ and $H_{P}(s)$. For the case of the ITER HF prototype magnetic sensors presented here, we do not consider the measurement and interpretation of $H_{D}(s)$ as we limit ourselves to analyzing the impedance as measured at the sensor's output.

For the case of the ITER HF prototype magnetic sensors, the probe-cable circuits consist simply of the probe itself plus a very short $(<10 \mathrm{~cm})$ screened twistedpair cable that brings the signal from the sensor to the impedance measurement device, in our case an HP impedance meter. The simplest circuit model that can reasonably account for the probe's (series) inductance and resistance, but neglects the cable except for its capacitance and admittance (parallel resistance), in parallel with the probe itself, is shown in Fig. A.1a. This is conceivable because the wavelength of a $1-\mathrm{MHz}$ signal is $300 \mathrm{~m}$, whereas the total length of the probe-cable system is at the most $\sim 8 \mathrm{~m}$. Note that the value of $1 \mathrm{MHz}$ is the highest Nyquist frequency for which one needs to design the ITER digitizers. Consequently, a model using conventional circuit elements is feasible. The circuit shown in Fig. A.1a can be generalized to include $k$ different cable sections, each having slightly different physical characteristics, which lead to the physics (and mathematics) of the generalized $k$ 'th-order circuit, as shown in Fig. A.1c. In all cases the measurement device at the output of the probe-cable section has a high impedance input ( $1 \mathrm{M} \Omega$ in the case of the impedance meter used in this work), which can then be neglected.

\section{A.I. IMPEDANCE OF THE MODEL CIRCUIT}

For the simple 1-pole circuit (model A in Fig. A.1a), the ac impedance is easily determined to be

$$
Z_{A}(i \omega)=\frac{R_{P}+s L_{P}}{1+\left(R_{P}+s L_{P}\right)\left(P_{1}+s C_{1}\right)}=\frac{N_{A}(i \omega)}{D_{A}(i \omega)},
$$

where $N_{A}(s)$ and $D_{A}(s)$ are polynomials in $s=i \omega$ representing the numerator and denominator of the impedance. The 2-pole circuit (model B) just consists of the 1-pole circuit, plus $R_{2}$ and $L_{2}$ in series and $C_{2}$ and $P_{2}$ in parallel, so the impedance becomes (after grinding out the relevant algebra): 
(a)

"Input"

$\left(V_{p}=N A d B / d t\right)$

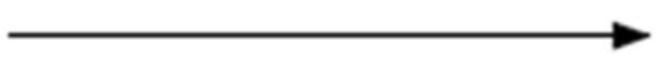

Output

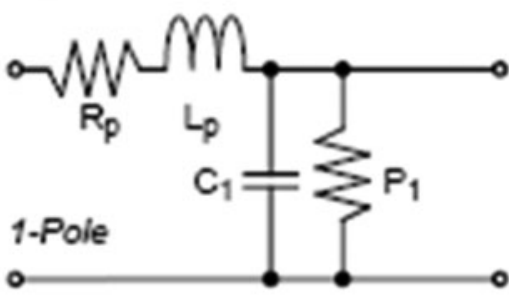

$\left(V_{\text {out }}\right)$

(b)

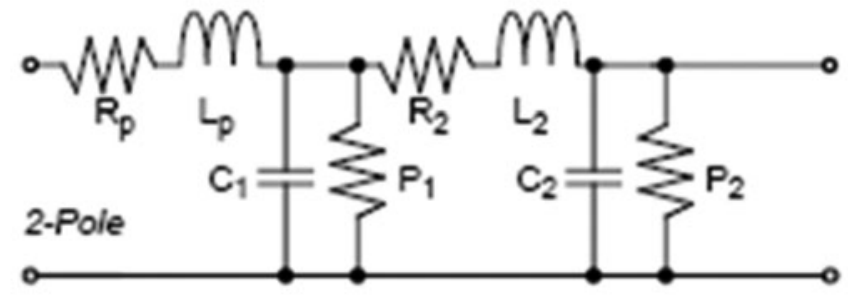

(c)

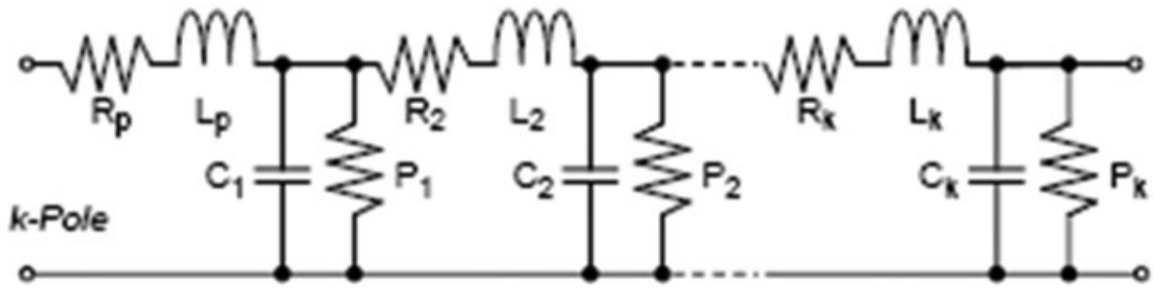

Fig. A.1. Schematics of magnetic probe circuit models. The "input" (driving voltage from $\partial B / \partial t$ ) is on the left, and the "output" (which goes to the front-end electronics) is on the right. The $P$ terms are for the admittances (parallel resistances, $P_{k}=1 / R_{\| k}$ ) of the transmission cable. Note that a more general formulation for these $P$ terms for the 2-pole onward model also includes a frequency dependency due to the parallel inductance of the transmission cables, $P_{k}(s)=1 /\left(R_{\| k}+s L_{\| k}\right)$, and it is this formulation that is used in this work. (a) Simple single-pole model (model A), which includes the probe and one set of elements for the effects of the cable. (b) Second-order model (model B), which has two sets of parameters for the cable. (c) Generalization of the circuit model to a $k$-pole system with an arbitrary number of circuit parameters (model K).

$Z_{B}(i \omega)=\frac{R_{2}+s L_{2}+Z_{A}}{1+\left(R_{2}+s L_{2}+Z_{A}\right)\left(P_{2}+s C_{2}\right)}=\frac{N_{B}(i \omega)}{D_{B}(i \omega)}$

Note that here $P_{2}=1 /\left(R_{\| 2}+s L_{\| 2}\right)$ for the sake of generality. It is now becoming clear that we are dealing with rational functions in $s$. Also, the order of the numerator and denominator polynomials increases with the number of poles in the circuit, and the number of circuit elements is four times the order of the circuit. Looking now to the $k$ 'th-order circuit, it should be clear that as the order of the circuit increases the algebra becomes increasingly unmanageable. However, recursion rules can be easily (but tediously) derived for how $Z$ changes as the order of the circuit increases. We can start with the rational function $Z_{k}(s)=N_{k}(s) / D_{k}(s)$, where $N_{k}(s)$ and $D_{k}(s)$ are the polynomials representing the numerator of the rational function and the denominator of the rational function, respectively. Then, in adding another segment, we simply hitch on $R_{k+1}$ and $L_{k+1}$ in series and $C_{k+1}$ and $P_{k+1}$ in parallel. To simplify the notation henceforth, let us define $X_{k+1}=P_{k+1}+s C_{k+1}$ and $Y_{k+1}=R_{k+1}+s L_{k+1}: X_{k+1}$ is now the parallel term and $Y_{k+1}$ the series term. Note that while each $X$ and $Y$ are both linear functions in $s, Y$ has units of $(\Omega)$ and $X$ has units of $(1 / \Omega)$. Then the ac impedance $Z_{k+1}(s)$ for the circuit of order $k+1$ becomes:

$$
\begin{aligned}
Z_{k+1}(s) & =\frac{R_{k+1}+s L_{k+1}+Z_{k}}{1+\left(R_{k+1}+s L_{k+1}+Z_{k}\right)\left(P_{k+1}+s C_{k+1}\right)} \\
& =\frac{N_{k+1}(s)}{D_{k+1}(s)} \\
\frac{N_{k+1}(s)}{D_{k+1}(s)} & =\frac{N_{k}(s)+D_{k}(s) Y_{k+1}(s)}{D_{k}(s)+\left[N_{k}(s)+D_{k}(s) Y_{k+1}(s)\right] X_{k+1}(s)} .
\end{aligned}
$$


Note that all these impedances $Z_{k}(s)$, as represented in Eqs. (A.3), (A.4), and (A.5), are calculated at the right terminal pair of the corresponding circuits shown in Fig. A.1, with the left side "numerically" shorted to have a closed electrical circuit. By inspection of Eq. (A.5) one can very quickly identify the recursion rules $N_{k+1}=N_{k}+$ $D_{k} Y_{k+1}$ and $D_{k+1}=D_{k}+X_{k+1} N_{k+1}$. It is paramount to remark here that the "only good" (i.e., correct in physics/ engineering terms) circuit model is given by a rational function in $s=i \omega$ with $\operatorname{deg}\left(N_{k}\right)=\operatorname{deg}\left(D_{k}\right)+1$ and $\operatorname{deg}(D)=2 k$, with $k$ being the number of poles (i.e., resonances) in the circuit.

Since the impedance is a rational function, it can be represented by two vectors in the linear space of polynomial functions (one for the numerator and one for the denominator). Furthermore, the process of adding series terms $\left(Y_{k}\right)$ and parallel terms $\left(X_{k}\right)$ to a given circuit (and thus transforming $Z=N / D$ via the recursion rules) can be represented by linear operators in this space. The elements of each operator are determined by the circuit elements that are added in each stage. To distinguish the linear-space representation from the physical circuit description, we can define $N_{j}=\mathbf{B}_{\mathbf{j}}$ and $D_{j}=\mathbf{A}_{\mathbf{j}}$ in the linear space, where (using a MATLAB-style polynomial notation, i.e., consistently with the code written to implement this system of equations):

$$
\mathbf{B}_{\mathbf{j}}=\left[b_{j+1}+b_{j} s+b_{j-1} s^{2}+\cdots+b_{1} s^{j-1}+b_{0} s^{j}\right]
$$

and

$$
\mathbf{A}_{\mathbf{j}}=\left[a_{j+1}+a_{j} s+a_{j-1} s^{2}+\cdots+a_{1} s^{j-1}+a_{0} s^{j}\right] .
$$

The process of polynomial multiplication is therefore the same as that of convoluting the vectors whose elements are the polynomial coefficients, so the recursion rules can be rewritten using the convolution matrices. Adding a series component (inductor-resistor pair) $Y_{k}=R_{k}+L_{k} s$ changes only the numerator of the impedance, with $\mathbf{B}_{\mathbf{j}+\mathbf{1}}=\mathbf{B}_{\mathbf{j}}+\mathbf{L}_{\mathbf{j}} \mathbf{A}_{\mathbf{j}}$ and, similarly, adding a parallel component (capacitance-admittance pair) $X_{k}=$ $P_{k}+C_{k} s$ has a satisfyingly symmetric effect, with $\mathbf{A}_{\mathbf{j}+\mathbf{1}}=$ $\mathbf{A}_{\mathbf{j}}+\mathbf{C}_{\mathbf{j}} \mathbf{A}_{\mathbf{j}}$, where the operators $\mathbf{L}_{\mathbf{j}}$ and $\mathbf{C}_{\mathbf{j}}$ are respectively given by:

$$
\mathbf{L}_{\mathbf{j}}=\left(\begin{array}{ccccc}
R_{k} & 0 & 0 & 0 & \ldots \\
L_{k} & R_{k} & 0 & 0 & \ldots \\
0 & L_{k} & R_{k} & 0 & \ldots \\
0 & 0 & L_{k} & R_{k} & \ldots \\
\ldots & \ldots & \ldots & \ldots & \ldots
\end{array}\right)
$$

and

$$
\mathbf{C}_{\mathbf{j}}=\left(\begin{array}{ccccc}
P_{k} & 0 & 0 & 0 & \ldots \\
C_{k} & P_{k} & 0 & 0 & \ldots \\
0 & C_{k} & P_{k} & 0 & \ldots \\
0 & 0 & C_{k} & P_{k} & \ldots \\
\ldots & \ldots & \ldots & \ldots & \ldots
\end{array}\right)
$$

By starting off with the null circuit $\mathbf{B}_{\mathbf{0}}=0$ and $\mathbf{A}_{\mathbf{0}}=1$ and then systematically adding circuit elements by applying the appropriate transformations $\mathbf{L}_{\mathbf{j}}$ and $\mathbf{C}_{\mathbf{j}}$, one can rapidly construct the rational impedance for the whole circuit. Because the operators are invertible, given a rational function for the impedance, one can extract the parameters of the circuit elements $L_{k}, R_{k}, C_{k}$, and $P_{k}$. From this set of data, we can then compute a $Z$-model via Eq. (A.5) and compare it with the actual measurements.

A slightly different physical and numerical approach to determine the equivalent circuit parameters is to consider the measured $Z$-data as an example of a generalized multipole resonant system (i.e., not necessarily representing a driving-point impedance measurement), for which then the transfer function can be given $a^{26}$ :

$$
\begin{aligned}
H(i \omega) & =\sum_{p=1}^{k} \frac{\alpha_{p}+i \beta_{p} \omega / \omega_{0, p}}{1+\left(\omega / \omega_{0, p}\right)^{2}\left(2 i \gamma_{p} / \omega-1\right)} \\
& =\frac{A_{(2 k+1)}(i \omega) \rightarrow A_{(2 k+1)+2 j}(i \omega)}{B_{2 k}(i \omega)}+D_{l}(i \omega),
\end{aligned}
$$

where $\alpha_{p}$ and $\beta_{p}$ are fit parameters and $\gamma_{p}$ and $\omega_{0, p}$ are the bandwidth frequency and self-resonance frequency, respectively, for each one of the poles $p=1 \rightarrow k$. Now a numerical fit can be performed, which can be expressed in terms of a rational function as $H(i \omega)=$ $A_{m}(i \omega) / B_{n}(i \omega)+D_{l}(i \omega)$, where $D$ represents a direct (nonresonant) coupling contribution, expressed as a polynomial in $i \omega$ of arbitrary $l$-order. The order of the denominator $B_{n}(i \omega)$ is fixed as $n=2 k$, whereas the order of the numerator $A_{m}(i \omega)$ can be in the range $m=$ $(2 k+1) \rightarrow(2 k+1)+2 j$, with $j$ as an integer number. This gives more numerical flexibility in modeling the circuit and sometimes helps in overcoming the computational difficulties that one may find when using a (numerically simple) ladder-type circuit model instead of the (more numerically complex, and never analytically solvable, although more exact in principle) distributedelements circuit model. Using recursive relations, one can then determine $L_{k}, R_{k}, C_{k}$, and $P_{k}$ for any pole in the resonant circuit by solving Eq. (A.8), and compute a second Z-model using Eq. (A.5), which can be compared with the measured $Z$-data. The comparison between these two sets of values for $L_{k}, R_{k}, C_{k}$, and $P_{k}$, 
the two Z-models and the experimentally measured $Z$-data, gives a very accurate assessment of the accuracy of the circuit model used for the analysis, hence giving confidence in the resulting transfer function, which is used for the calibration of the HF magnetic measurements.

\section{A.II. RELATIONSHIP BETWEEN MEASURED IMPEDANCE AND DEDUCED TRANSFER FUNCTION}

In principle, if one has a measurement of the circuit impedance as a function of frequency, plus a knowledge of the circuit structure (though not necessarily the values of the components), one can extract the transfer function between different parts of the circuit. In this case, we are interested in the ac component of the transfer function $H_{A C}(s \neq 0)$, and we then use a separately deduced value for the geometrical value of $(N A)_{E F F}(s=0)$ to determine the dc gain of the magnetic sensor; i.e., we set that $H_{P}(s)=s \times(N A)_{E F F}$ $(s=0) \times H_{A C}(s)$. The "hard way" to determine $H_{P}(s)$ is to use the inverses of the circuit transformations described above to determine all of the component values from a polynomial fit to the impedance and then construct the transfer function from the known circuit structure. This is not a simple problem and is likely to be fraught with numerical error if the impedance measurement or fit is imperfect, or if certain circuit elements have small impedances.

A much more direct method is available for the class of circuits of interest here, because there is a simple and direct relationship between the rationalized impedance $Z_{k}(s)=N_{k}(s) / D_{k}(s)$ and $H_{P, k}$ : for circuits of the form represented in Fig. A. 1 of any order $k, H_{P, k}=1 / D_{k}$. This theorem is proven in Ref. 4 for $H_{P, k}$, which includes the contribution of $s \times(N A)_{E F F}(s)$, but it is straightforward to see from the derivation presented there that this proof is also valid for any circuit for which the impedance can be represented as a rational function in $s$. In other words, if the circuit impedance $Z$ is represented as a rational function in $s, Z(s)=N(s) / D(s)$, then the ac transfer function $H_{A C}(s)$ from input to output is simply and always $H_{A C}(s)=1 / D(s) /\left(s \times(N A)_{E F F}(s=0)\right)$, which is a very useful result. This is absolutely fundamental to this ac calibration technique because it implies that one can simply measure the ac circuit impedance, find the lowest-order rational function that fits it, cast it into an appropriate ratio of two polynomials, and then use the denominator as the transfer function, with no additional work. Furthermore, the rational fit can be verified by taking the fitted impedance and extracting the values of the parameters of the circuit to see if they are reasonable.

As a final note, the reader is reminded that the mathematical formulation employed here is slightly different from that defined in Ref. 4, where the numerical procedure is related to interpreting $H_{P}(\omega)$ as deduced from $Z(\omega)$, whereas here we deal with interpreting $H_{A C}(\omega)$ as deduced from $1 / D(s) /\left(s \times(N A)_{E F F}(s=0)\right)$.

\section{A.III. RELATIONSHIP BETWEEN MEASURED IMPEDANCE AND FREQUENCY-DEPENDENT EFFECTIVE AREA}

The effective area $(N A)_{E F F}$ is usually seen as a dc gain for the actual voltage measured at the $\mathrm{HF}$ pickup coil; hence, it is often solely determined independently from the impedance measurements using a Helmholtz coil system at low frequencies, usually below $1 \mathrm{kHz}$ or even less. On the other hand, when comparing Eqs. (A.1) and (A.2), it is obvious that $H_{P}(\omega)=i \omega \times(N A)_{E F F}(\omega)$ if we consider the voltage measurement $V_{M E A S}$ before any digitization equipment. We now take the ansatz that the frequency-dependent $(N A)_{E F F}(\omega)$ can be separated in a dc gain $(N A)_{E F F}(\omega=$ $0)$, which corresponds to the geometrical value of the effective area, and a frequency-dependent transfer function $H_{A C}(\omega)$, which can be deduced from the ac impedance measurements:

$$
\begin{aligned}
V_{M E A S}(\omega) & =H_{P}(\omega) \times B_{M E A S}(\omega) \\
& =\left[(N A)_{E F F}(\omega=0) \times H_{A C}(\omega) \times i \omega\right] B_{M E A S}(\omega) .
\end{aligned}
$$

Hence, from Eq. (A.9) we derive that $H_{P}(\omega)=(N A)_{E F F}$ $(\omega=0) \times H_{A C}(\omega) \times i \omega$. For the single-pole circuit model of Fig. A.1, if the frequency range of the impedance and effective area measurements overlap, i.e., the Helmholtz coil measurements are taken at sufficiently high frequencies $\omega>\omega_{H}$, it is straightforward to construct an equivalent $(N A)_{E F F}(\omega)$ as:

$$
\begin{aligned}
(N A)_{E F F}(\omega)= & (N A)_{E F F}^{\text {geom }}(\omega=0) \\
& \times\left|\frac{1}{1+(R+i \omega L)(P+i \omega C)}\right| \\
= & (N A)_{E F F}^{\text {Helmholtz }}(\omega),
\end{aligned}
$$

where $(N A)_{E F F}(\omega=0)$ is the geometrical value of the effective area and $R, L, C$, and $P$ are determined from fitting the measured $Z$-data for the single-pole circuit, and this calculation must agree with the measurements made with the Helmholtz setup for frequencies above a cutoff value $\omega>\omega_{H}$. This provides a conclusive confirmation of the validity of the transfer function obtained from the impedance measurements, at least in some 
frequency range. Based on our previous direct measurement experience on the JET HF magnetic sensors, one can take as a practical value for $\omega_{H}$ the frequency point where the phase of $Z$ is no longer constant (i.e., the sensor is not anymore a "pure dc resistor," so that $\left.R \gg L \omega_{H}\right)$ but starts increasing as a linear function of $\omega$ (i.e., the sensor behaves as an inductor in series with a smaller dc resistance) and $\operatorname{arctg}(\omega L / R)>20 \mathrm{deg}$. Depending on the actual values of the self-inductance and dc resistance of the sensor, the value of $\omega_{H}$ can range between $\sim 1 \mathrm{kHz}$ and $\sim 20 \mathrm{kHz}$.

Finally, another estimate of the frequency-dependent effective area can be obtained by comparing the values of the ac (i.e., derived from the impedance measurements) and dc (i.e., derived using the geometry of the sensor) inductance, since by definition we have that $L(\omega)=$ $d \phi_{T O T}(I, \omega) / d I(\omega)$, where $\phi_{T O T}(I, \omega)$ is the total magnetic flux produced by the current $I(\omega)$ and linked through the effective area of the magnetic sensor, irrespective of its current source. Since $\phi_{T O T}(I, \omega)$ can be computed directly using a nominal current element $d I(\omega)$ and the exact geometry of the 3-D sensor's solenoidal winding, the solution of the Ampère's law provides another estimate for $(N A)_{E F F}(\omega)$.

\section{A.IV. SIMPLE DETERMINATION OF DC EQUIVALENT CIRCUIT PARAMETERS}

The analysis starts from identifying numerically the sensor's resonant frequency $\omega_{0}=1 /(L C)^{1 / 2}$ and bandwidth $\gamma=\left(\omega_{0}\right)^{2}(R C+P L) / 2$, where $R, L, C$, and $P$ are the resistance, inductance, capacitance, and parallel admittance of each test HF magnetic sensor, respectively. Then, for frequencies such that the sensor (plus cable) can be considered essentially as a pure inductor (i.e., for frequencies such that $70<$ phase $(Z)(\mathrm{deg})<110)$, we have that $\operatorname{imag}(Z) \sim \omega L$, hence, an average $L$ can be computed over this frequency range, which then allows one to determine $C$ from the sensor's resonant frequency. These values of $L$ and $C$ are verified against the corresponding results obtained in the frequency range where the HF magnetic sensor can be considered almost as a pure capacitor (i.e., for frequencies such that $-110<$ phase $(Z)(\operatorname{deg})<-70)$, where we have, correspondingly, that imag $(Z) \sim-1 /(\omega C)$. A very good agreement is usually found between these two different estimates of $L$ and $C$, and the average value can be taken. Using these values of $L$ and $C$ and assuming a fixed (i.e., frequencyindependent) $P$, as given by the measurement of the $\mathrm{dc}$ isolation resistance, $R$ can then be determined from the sensor's quality factor $Q=\omega_{0} /(2 \gamma)$. The best fit to the measured $Z$-data is then constructed from the numerical procedure giving the circuit parameters described above. The best-fit impedance is then finally evaluated using the formula:

$$
\begin{aligned}
Z(\omega)= & \frac{R+i \omega L}{1+(R+i \omega L)(P+i \omega C)} \\
= & \frac{R+i \omega L}{(1+P R)-\left(\frac{\omega}{\omega_{0}}\right)^{2}\left(1-i \tau \frac{\omega_{0}^{2}}{\omega}\right)} \\
= & \frac{\alpha+i \beta \frac{\omega}{\omega_{0}}}{(1+P R)-\left(\frac{\omega}{\omega_{0}}\right)^{2}\left(1-2 i \frac{\gamma}{\omega}\right)},
\end{aligned}
$$

where the parameters $\alpha, \beta, \omega_{0}$, and $\gamma$ are determined from the numerical fit and $R, L, C$, and $P$ are the model circuit parameters, with $\tau=(R C+P L)=2 \gamma\left(\omega_{0}\right)^{2}$.

\section{ACKNOWLEDGMENTS}

This work was partly supported by the European Communities under Contracts of Association and was partly performed within the framework of the European Fusion Development Agreement (EFDA) under Tasks TW4-TPDS-DIASUP, TW5TPDS-DIASUP, TW6-TPDS-DIADES, and TW6-TPDSDIADEV. The views expressed in this publication are the sole responsibility of the authors and do not necessarily reflect the views of Fusion for Energy (F4E) or the European Commission. We would like to acknowledge the discussions we have had during this work with current members of the ITER Organization (A. Encheva, G. Vayakis, C. Walker) and of EFDA and F4E (S. Arshad, C. Ingesson). We also would like to acknowledge N. Lam (JET) for useful discussions about his experience with the manufacturing and assembly problems encountered with the JET HF pickup coils.

\section{REFERENCES}

1. G. VAYAKIS et al., Rev. Sci. Instrum., 74, 2411 (2003).

2. E. J. STRAIT, E. D. FREDRICKSON, J-M. MORET, and M. TAKECHI, Fusion Sci. Technol., 53, 304 (2008); see also references therein; see also A. J. H. DONNÉ et al., "Chapter 7: Diagnostics," Nucl. Fusion, 47, S337 (2007); see also references therein; see also D. TESTA et al., IEEE Trans. Plasma Sci., 38, 3, 284 (2010).

3. G. VLAD et al., Plasma Phys. Control. Fusion, 46, 1 (2006); see also S. PINCHES et al., Nucl. Fusion, 46, S904 (2006); see also M. P. GRYAZNEVICH and S. E. SHARAPOV, Nucl. Fusion, 46, S942 (2006).

4. R. F. HEETER, A. FASOLI, S. ALI-ARSHAD, and J-M. MORET, Rev. Sci. Instrum., 71, 11, 4092 (2000).

5. S. GUENTER et al. Nucl. Fusion, 47, 920 (2007).

6. M. J. HOLE and L. C. APPEL, Plasma Phys. Control. Fusion, 51, 045002 (2009). 
7. E. J. STRAIT, Rev. Sci. Instrum., 77, 023502 (2006); see also references therein.

8. K. SHINOHARA et al., Plasma Phys. Control. Fusion, 46, S31 (2004); see also references therein.

9. J. A. SNIPES, ITER Organization, Plasma Operation Group, Personal Communication.

10. G. VAYAKIS, Personal Communication (Design Description Document 55.A0.00, Section 6.1.7, ITER document reference: 3UYQGX v1.5).

11. International Tokamak Physics Activity Working Group Mtg Energetic Particles MHD Control, Seoul, South Korea, October 18-20, 2010: http://itpa2010.snu.ac.kr/ (current as of Sep. 28, 2011).

12. D. TESTA, H. CARFANTAN, R. CHAVAN, J. B. LISTER, J-M. MORET, and M. TOUSSAINT, Fusion Sci. Technol., 57, 208 (2010); see also D.TESTA, H. CARFANTAN, R. CHAVAN, J. B. LISTER, J-M. MORET, and M. TOUSSAINT, Fusion Sci. Technol., 57, 238 (2010); see also D.TESTA et al., "Assessment of the ITER High-Frequency Magnetic Diagnostic Set," Fusion Eng. Des., 86, 1149 (2011), doi:10.1016/ j.fusengdes.2011.03.118.

13. J. GERNHARDT, "Magnetic Diagnostic on ASDEX Upgrade with Internal and External Pick-up Coils," IPP1/262, Garching (1992).

14. "Progress in the ITER Physics Basis," Nucl. Fusion, 47, (2007).

15. A. ENCHEVA et al., Final Report for the EFDA task TW5DIASUP; see also A. ENCHEVA et al., "Progress on the Design of Various Magnetic Sensors for ITER," presented at 10th
ITPA Topl. Group Mtg. Diagnostics, Moscow, Russia, April 10-14, 2006

16. ITER_D_222QLG, V3.1, ITER Load Specifications, Annex to PID (Apr. 2006).

17. M. ROCCELLA et al., "Detailed EM Analyses of ITER Components," European Fusion Development Agreement Contract 01-634, 2003.

18. ITER Material Properties Handbook, ITER Document G74MA16.

19. H. LI and X. ZENG, "Study on the Structure and Properties of Thick-Film Capacitors Fabricated by Laser MicroCladding and Rapid Prototype," J. Mater. Processing Technol., 184, 184 (2007).

20. M. TOUSSAINT et al., "Design of the ITER HighFrequency Magnetic Diagnostic Coils," Fusion Eng. Des., 86, 1248 (2011), doi:10.1016/j.fusengdes.2011.03.080.

21. D. TESTA et al., Fusion Sci. Technol., 59, 376 (2011).

22. H. TAKAHASHI, S. SAKAKIBARA, Y. KUBOTA, and H. YAMADA, Rev. Sci. Instrum., 72, 3249 (2001).

23. G. ChitARIN, R. DElOGU, A. GAllo, and S. PERUZZO, Fusion Eng. Des., 84, 593 (2009).

24. D. TESTA et al., "Baseline System Design and Prototyping for the ITER High-Frequency Magnetic Diagnostic Set," Proc. 23rd Symp. Fusion Engineering, San Diego, California, May 30-June 5, 2009, doi:10.1109/fusion.2009.5226481.

25. J-M. MORET et al., Rev. Sci. Instrum., 69, 2333 (1998); see also Ref. 3 cited therein.

26. A. FASOLI et al., Phys. Rev. Lett., 75, 4, 645 (1995). 